\title{
THE EFFECTS OF SCRAMBLING EPISODE COMPONENTS ON MEMORY FOR A PICTURE STORY: NOT UNDERSTANDING, BUT RECOGNIZING WHAT YOU SAW
}

by

\section{ADAM MICHAEL LARSON}

B.S., Iowa State University, 2006

\begin{abstract}
A THESIS
submitted in partial fulfillment of the requirements for the degree
\end{abstract}

MASTER OF SCIENCE

Department of Psychology

College of Arts and Sciences

\section{KANSAS STATE UNIVERSITY \\ Manhattan, Kansas}

2010

Approved by:

Major Professor

Dr. Lester C. Loschky 


\section{Copyright}

ADAM MICHAEL LARSON

2010 


\begin{abstract}
How are episodes of picture stories remembered, and what role does the order of their components (exposition, complication, and resolution) play in that memory? We presented picture sequence episodes of the "Red Balloon" with the order of their components either normal or scrambled. As predicted by story grammar theories, scrambling episode components reduced self-rated comprehension and recall. However, scrambling also produced faster recognition memory responses for hits. This suggests that episode component scrambling interfered with the transformation of perceptual to conceptual information in LTM, producing an advantage for familiarity over recollection. Additionally, recall memory decreased monotonically from exposition to resolution, whereas recognition memory showed the opposite result, and this was the same whether components were normally ordered or scrambled. This suggests that memory for picture story episode components is based on their information content rather than their temporal order, and that information from picture stories moves from perceptual to conceptual memory representations.
\end{abstract}




\section{Table of Contents}

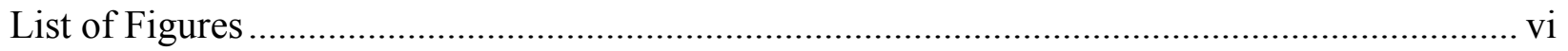

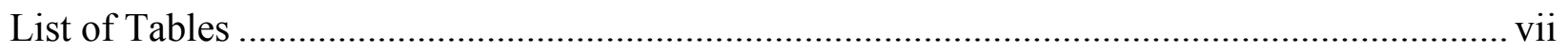

Acknowledgements ................................................................................................... vii

CHAPTER 1 - Memory for Picture Stories ............................................................................ 1

Theoretical Perspectives on Story Structures ........................................................................ 3

Predictions for Story Scrambling on Memory ..................................................................... 7

Predictions for Episode Component Memory ........................................................................... 9

The Representation of Events in Episode Components............................................................ 12

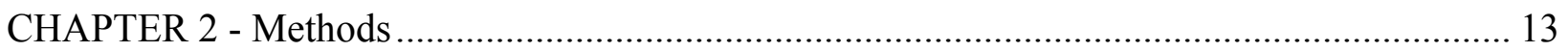

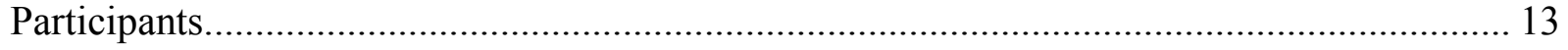

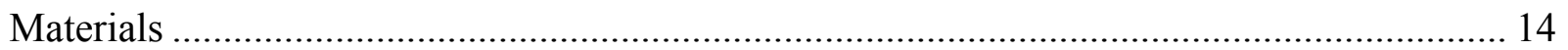

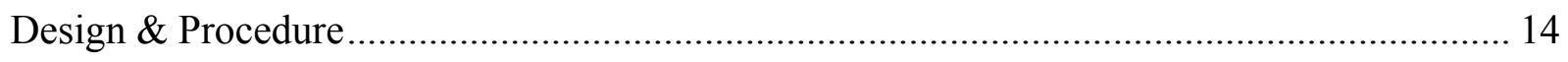

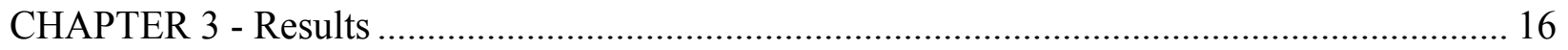

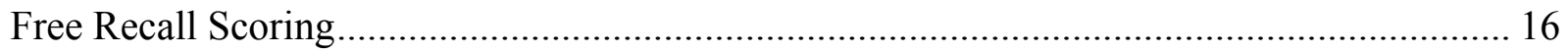

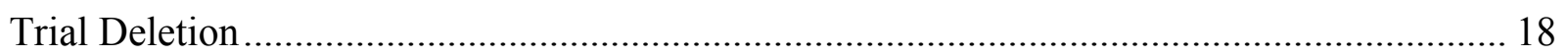

Effects of Story Scrambling on Memory and Comprehension................................................ 19

Self-Rated Comprehension and Recall Memory ................................................................ 19

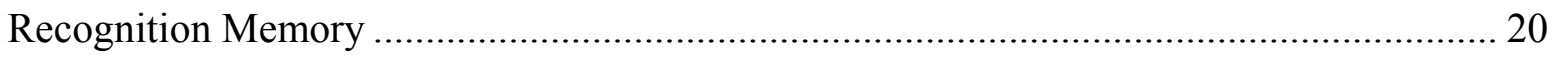

Episode Component Memory ………………………........................................................ 21

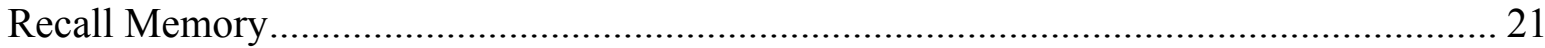

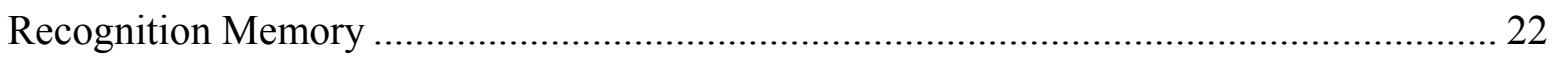

The Serial Position Hypothesis vs. Component Information Hypothesis ................................ 24

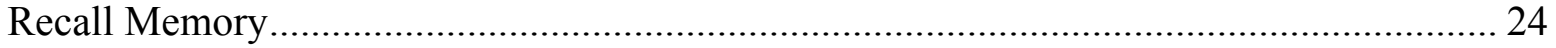

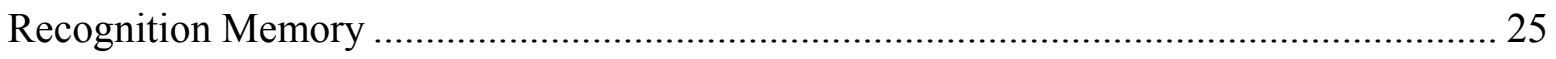

The Representation of Events in Episode Components........................................................ 26

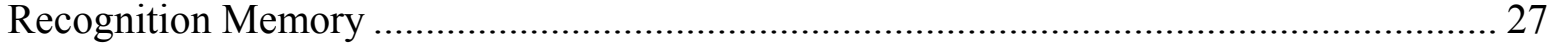

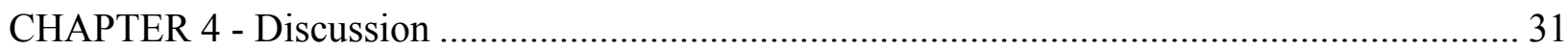

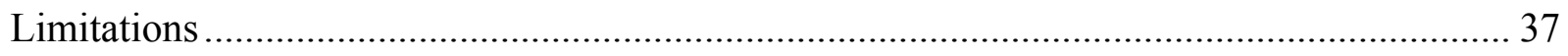

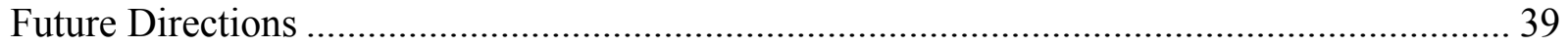




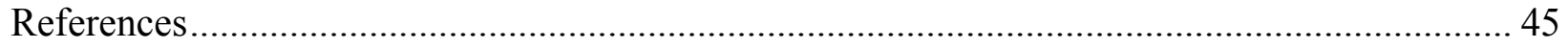

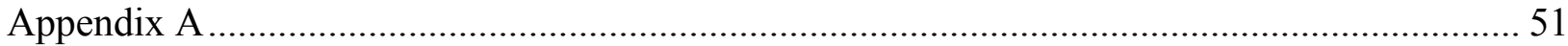

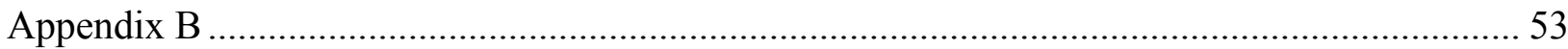




\section{List of Figures}

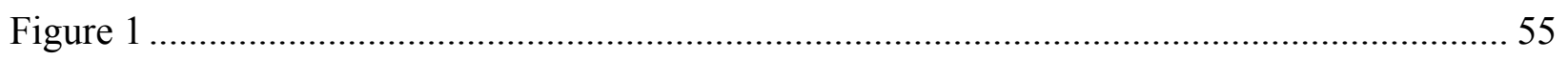

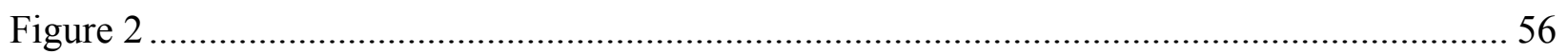

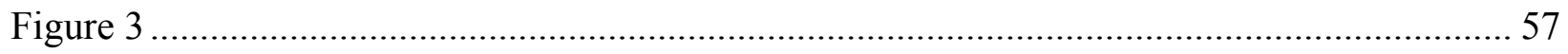

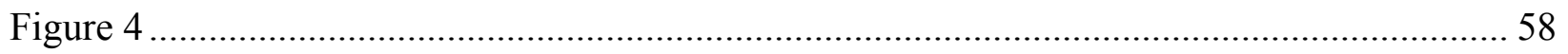

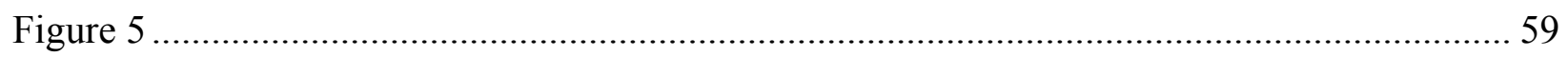

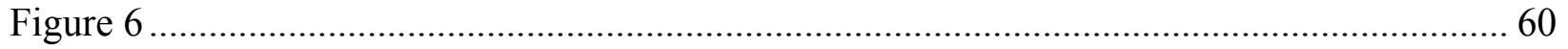

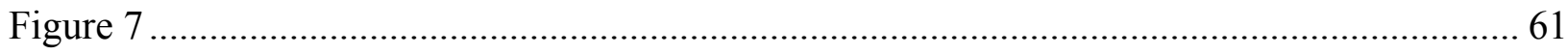

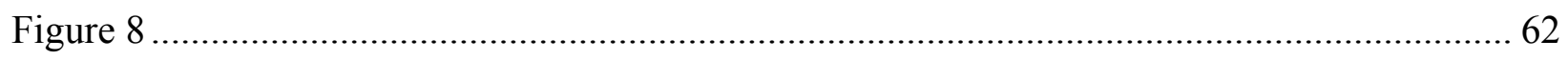

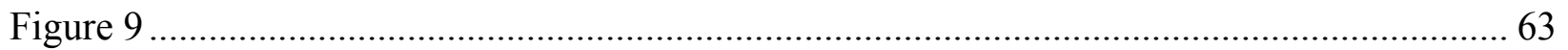




\section{List of Tables}

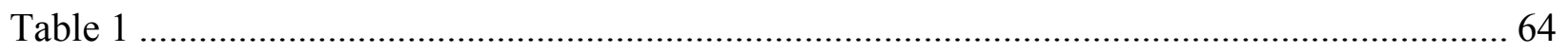

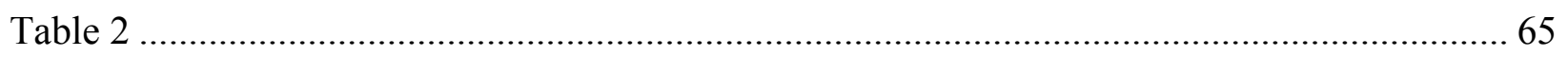

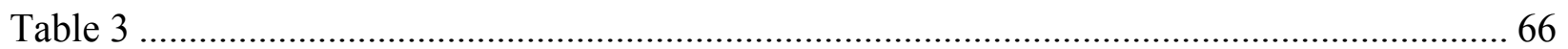

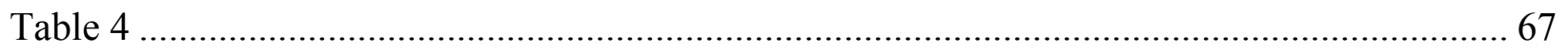

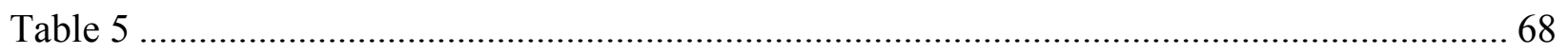

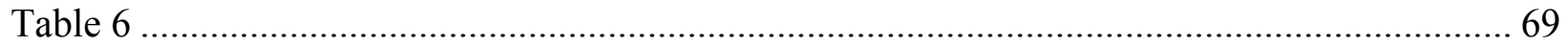

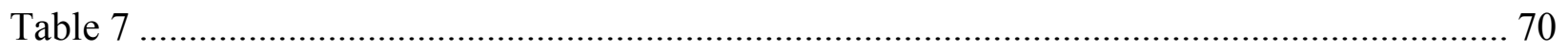

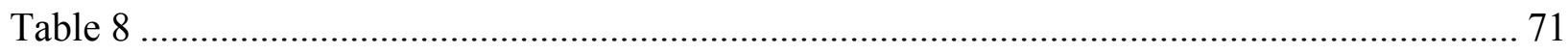

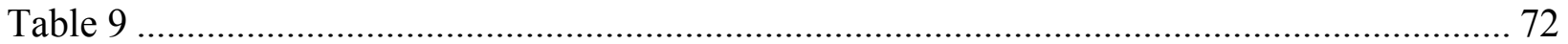

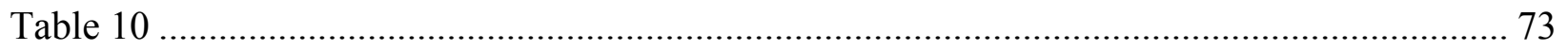

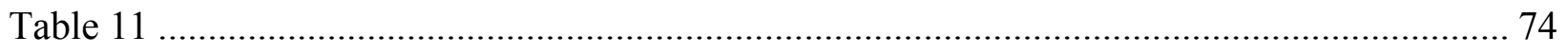

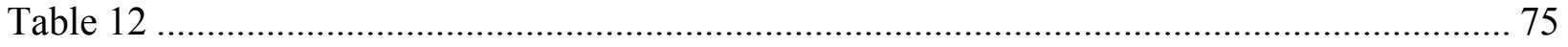

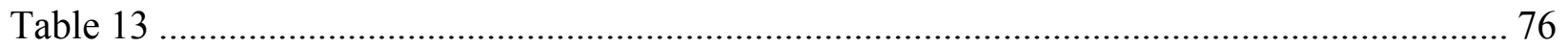

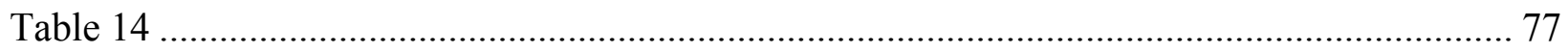




\section{Acknowledgements}

I want to give many thanks to my advisor Dr. Lester Loschky for his continuous support and dedication since the moment I arrived at Kansas State. I also want to thank my fellow graduate students Bernardo de la Garza and Tyler Freeman for all of their helpful comments and feedback they have given me on this project. I am very humbled by the dedication of Chris Wallace and Susie Goddard who helped me code the data presented in this thesis. Additionally, I thank all of the members of Dr. Loschky's Visual Cognition lab, including Ryan Ringer, Josh Davis, Caroline Kridner, and Allison Klinock who helped me collect the data for this thesis.

Finally, I would like to thank the members of the committee, Drs. Lester Loschky, Richard Harris, and Donald Saucier, who helped shape this project into what it is today. 


\section{CHAPTER 1 - Memory for Picture Stories}

The Effects of Scrambling Episode Components on Memory for a Picture Story: Not Understanding, But Recognizing What You Saw

From the moment that we open our eyes in the morning to moment that we close them at night, we perceive our everyday lives as a flowing and continuous experience. For instance, after waking up, you may decide to walk to the kitchen and make breakfast while watching the morning news. Therefore, we would expect that any abrupt change in our visual experiences would be extremely difficult to comprehend, for example, if when entering the kitchen for breakfast, you find yourself sitting at your desk at work. However, we manage to comprehend such large changes in setting, characters, or situations when going from page to page in a picture story, frame to frame in a comic book, or shot to shot in a film. Our ability to comprehend these changes we see in picture stories should be related to our ability to understand events in real life. Therefore, by understanding how we perceive, understand, and remember such events in picture stories, we may better understand how our brain comprehends and remembers information in our everyday lives.

Picture stories present viewers with a tremendous amount of information including the introduction of the setting and the characters, the presentation of a goal, and interactions between characters in attempts to reach the goal. For example, Figure 1 shows pictures from an episode from the movie "The Red Balloon" (Lamorisee, 1956).

\section{[[Insert Figure 1 here $]$}

In order to comprehend the story, the viewer begins by encoding perceptual information in the images, like the character (e.g., the boy), objects (e.g., a balloon and a light pole), and their spatial relationships (e.g., the boy is below the balloon, which is on top of a light pole). The 
perceptual information is then conceptualized as semantic information, like "the boy climbs a light pole to get a balloon." Such, comprehension processes are influenced by top-down information. For instance, the viewer has a schema for the organization of a story that allows them to map the information that is seen onto the story schema (Mandler \& Johnson, 1977; Thorndyke, 1977). This creates expectations of what will happen next in the story. For instance, in the last image in the exposition, the boy looks up, as if he notices something, which creates the expectation that he may try to interact with that object. After viewing the entire story, the viewer can retrieve story information from long-term memory based on the expectations created from the story schema (Brewer \& Dupree, 1983).

A story schema can be decomposed into separate components, as described by story grammar theories (Kintsch, 1977; Mandler \& Johnson, 1977; Thorndyke, 1977; van Dijk, 1977). A story grammar is hierarchically organized into abstract levels. The entire story can be divided into episodes which are sets of actions intended to achieve a goal (Lichtenstein \& Brewer, 1980; Mandler \& Johnson, 1977; Thorndyke, 1977). Although various story grammar structures have been proposed (Kintsch, 1977; Mandler \& Johnson, 1977; Thorndyke, 1977; van Dijk, 1977), a commonly proposed structure is one in which each episode is divided into three sequentially ordered episode components called the exposition, the complication, and the resolution (Kintsch, 1977; Mandler \& Johnson, 1977). First, the exposition introduces the characters and the setting of the story. Second, an obstacle or goal is introduced to a character in the complication, and finally the problem is solved in the resolution. For instance, in the exposition presented in Figure 1, a small boy is walking in a city, and then goes down a flight of stairs, when he notices something above him on a light pole. In the complication, the boy tries to determine how to climb the pole and begins to do so. Finally in the resolution, the boy grabs a red balloon, climbs 
down the pole, and continues walking down another flight of stairs. Each episode component contains a causal link to the next component in the episode. Information connected by causal links in the story result in better memory compared to temporal links or links indicating simultaneous actions in the story (Mandler \& Johnson, 1977).

According to story grammar theories (Mandler \& Johnson, 1977; Thorndyke, 1977) when the episode component order is normal, memory for the story should be best. However, some of the story information will still be lost in long term memory (LTM). Specifically, decreasing recall is found across these sequentially ordered episode components (Baggett, 1979; Mandler \& Johnson, 1977), with the best recall for the exposition, and worst recall for the resolution. Interestingly, story grammar theories have not provided an explanation for this memory pattern across episode components. However, some potential explanations have been offered by theories of memory for text, pictures, and video.

\section{Theoretical Perspectives on Story Structures}

Dividing picture stories into separate structures (e.g., episodes or components) is very similar to segmenting written stories, or everyday events (Baggett, 1979; Gernsbacher, Varner, \& Faust, 1990; Magliano, Miller, \& Zwaan, 2001; Zacks, Speer, \& Reynolds, 2009). Picture stories and everyday activities can be segmented into structures referred to as events (Zacks \& Tversky, 2001). An event involves a specific object, character, or goal that has a specified beginning and ending. We can speculate as to a few hypothetical events from the picture story presented in Figure 1 (though these have not been empirically tested). The exposition may be represented by two events, consisting of the boy walking in a city (image 1 and 2), followed by the boy walking down the stairs (image 3 and 4). The complication may be represented by one event where the boy climbs a light pole to grab a balloon. The resolution may contain the final 
event where the boy climbs down the light pole and walks away. Alternatively, the complication and resolution might both contain an overlapping event, in which the boy climbs up the light pole to get the balloon and climbs down afterward. Segmenting events can occur within videos (Carroll \& Bever, 1975; Hard, Tversky, \& Lang, 2006; Newston 1973), a series of static scene images in a picture story (Gernsbacher, 1985; Gernsbacher, Varner, \& Faust; 1990), and written text (Baggett, 1979; Gernsbacher, 1985; Speer \& Zacks, 2005; Zacks, Speer, \& Reynolds, 2009; Zacks, Tversky, \& Iyer, 2001). Event Segmentation Theory provides an explanation for the perception of these discrete events in vision, through the interaction of both bottom-up and topdown processes (Reynolds, Zacks, \& Braver, 2007; Zacks, Speer, Sallow, Braver, \& Reynolds, 2007; Zacks, Tversky, \& Iyer, 2001).

According to Event Segmentation Theory (Reynolds, Zacks, \& Braver, 2007; Zacks, et al., 2007; Zacks, Tversky, \& Iyer, 2001) the process of event perception begins with the observer gathering low-level perceptual information from the environment. This information is processed and given semantic labels, and is used to construct an event model for the perceived event. Event models are working memory representations of what is currently happening. The construction of the event model is also influenced by top-down schemas from long-term memory for similar experiences, like the character's goals. When an accurate event model has been constructed, it can then be used to make a perceptual prediction regarding what will occur next. The prediction based on the event model is then compared to what later occurs. If the event model is accurate in its predictions, then the observed event is perceived as still continuing. However, if the number or qualitative severity of errors made by the event model reaches a threshold, the observed event is perceived as having ended because the event model is no longer predictive of the current event, thereby indicating that a new event model needs to be created. Greater cognitive 
processing occurs at the beginning of the event (i.e., the event boundary), which results in strong long-term memory representations. However, once the new event model is created, cognitive processing diminishes over the remainder of the event (i.e., the non-event boundary), resulting in worse memory compared to memory at event boundaries (Schwan \& Garsoffky, 2004; Swallow, Zacks, \& Abrams, 2009).

Event segmentation occurs passively, without conscious awareness (Speer, Zacks, \& Reynolds, 2007). Events can be segmented at either a coarse-grained or fine-grained level (Hanson \& Hirst, 1989; Kurby \& Zacks, 2007; Newston, 1973, Newston \& Engquist, 1976; Zacks, Tversky, \& Iyer, 2001). Event segmentation is hierarchically organized, with fine-grain events nested within coarse-grained events (Zacks, Kumar, Abrams, \& Mehta, 2009; Zacks, Tversky, \& Iyer, 2001). For example, a baseball game may be considered a coarse-grained event that is composed of nine innings or finer-grained events. Regarding the current study, an episode may be hypothesized to be a coarse-grained event that is composed of three finer-grained events or episode components. Research has shown that fine-grained segmentation produces greater recall (Hanson \& Hirst, 1989) and recognition memory (Lassiter, 1988; Lassiter, Stone, \& Rogers, 1988) than coarse-grained segmentation. This is consistent with the claim of Event Segmentation Theory that information that is segmented into finer events produces a greater number of event boundaries, which are encoded to a greater depth than at non-boundaries. Additionally, perceptual features like movement are more strongly correlated with fine-grained event boundaries, while conceptual features, like the goals' of an individual, seem to be related to coarse-grained event boundaries (Zacks, Kumar, Abrams, \& Mehta, 2009; Zacks \& Tversky, 2001). This suggests that one's segmentation style affects the features they monitor. 
The Structure Building Framework has been proposed as a theory of comprehension of text and picture stories (Gernsbacher, 1985; 1990), and is somewhat similar to Event Segmentation Theory. According to the theory, the comprehension process begins by reading a sentence or viewing a picture, which activates memory nodes (Gernsbacher, 1985; 1997). The information contained within these nodes lays the foundation for a structure where future information will be mapped onto it. This initial stage requires greater use of cognitive resources, resulting in longer reading times for the first sentence of a paragraph, story episode, or longer viewing times for the first picture of a new episode (Gernsbacher, 1983; Haberlandt, 1984; Haberlandt, Berian, \& Sandson, 1980). Retrieving information from memory is better when cued by presenting the first sentence of an episode than when cued by subsequent sentences (Mandler \& Goodman, 1982). Additional information is then mapped onto the structure. If the new information activates similar memory nodes nearby, then it should be mapped onto the current structure. Support for this hypothesized mapping function has been shown by the fact that a second sentence is read faster than the first when it is coherent with the first sentence, and therefore would be assumed to be mapped onto the same structure (Gernsbacher, 1990). This same effect has been shown at the episode component level. Specifically, reading times were longest at the beginning of a story episode, suggesting that information presented after the start of an episode was mapped onto the same structure (Haberlandt, Berian, \& Sandson, 1980). However, if new information presented lacks coherence (e.g., referential, temporal, spatial, or causal) with the initial sentence or picture, then the construction of a new substructure must begin due to the activation of a separate memory node. This process of constructing new structures is referred to as a processing shift, since one is shifting cognitive resources to construct a new structure. Processing shifts in Structure Building Framework are similar to event 
boundaries in Event Segmentation Theory (Zacks et al., 2007). Additionally, information from the previous structure (or event) is more difficult to access compared to information from the current structure (or event)(Gernsbacher, 1985; Swallow, Zacks, \& Abrams, 2009).

An alternative explanation is that an episode may be represented in memory by planschemas. These schemas use the inferred goals of a character to organize and retrieve action information (Brewer \& Dupree, 1983; Lichtenstein \& Brewer, 1980). Similar to Event Segmentation Theory, these goals and sub-goals are hierarchically organized by a causal "inorder-to" relationship. For instance, a character must first satisfy a sub-goal prior to completion of the goal. In Figure 1, if the boy's goal was to "get the balloon" then he would first have to climb the light pole in-order-to get the balloon. Based on the organization of the plan-schema, Lichenstein and Brewer (1980) found that recall for the goal was better than the sub-goal actions. Additionally, actions that were part of a plan-schema were better recalled than actions not part of a plan-schema (Brewer \& Dupree, 1983; Lichenstein \& Brewer, 1980). This suggests that the encoding and retrieval of event information is influenced by the hierarchical goal and sub-goal structure.

\section{Predictions for Story Scrambling on Memory}

The current study examines both recall and recognition memory for episode components in picture stories as a function of their order. First, what is the effect of scrambling the order of episode components in a picture story? Some research has shown that scrambling produces worse memory (Gernsbacher, 1985; Gernsbacher, Varner, \& Faust, 1990), while other research has not found such an effect (Kraft \& Jenkins, 1977; Kintsch, Mandel, \& Kozminsky, 1977). In addition, if a story is scrambled, it may be interpreted as consisting of numerous, discrete, finegrained events, which should result in more event boundaries and subsequently better memory. 
According to story grammar theory, episode components are linked by causal relationships, where the completion of one action allows for the completion of a second. Causal relationships provide a structure for integrating information presented in a story in memory, which has implications for information retrieval (Kintsch, Mandel, \& Kozminsky, 1977; Mandler \& Johnson, 1977; Thorndyke, 1977). When episode components conform to the ideal episode organization (i.e., the exposition first, followed by the complication, and then the resolution), the causal relationships between sequential components remain intact, which should produce better memory. However, if the organization deviates from the ideal structure, the causal connections between episode components will be replaced by weaker simultaneous or temporal relationships between episode components, resulting in worse recall (Mandler \& Johnson, 1977; Thorndyke, 1977). For instance, Thorndyke (1977) has shown that if a story's theme, defined as the goal for the main character, was in its normal position in the story, then both recall and recognition was best. However, recall and recognition memory decreased if the theme was placed at the end of the story, and decreased further if it was omitted from the story completely. Furthermore, both recall and recognition memory was worst when the sentences of the story were scrambled, independent of the placement of the theme in the story. For picture stories, Gernsbacher, et al. (1990) showed that if the pictures were scrambled, recognition for the surface level features (i.e., the image's left/right orientation) was worse compared to pictures in the normal order.

Nevertheless, consideration of Event Segmentation Theory suggests an interesting alternative prediction regarding the effects of story scrambling on memory. If episode components are scrambled, then it may become more difficult to monitor the character's goals, thus making it difficult to predict incoming perceptual information based on one's current event model. For this reason, it may become more important to monitor perceptual information in 
order to update one's event model. Thus, when the episode components are scrambled, one would be more likely to create more events resulting in better memory for that information compared to the normal order.

A third possibility suggests that there would be no difference between the scrambled and normal orders. For example, Lichtenstein and Brewer (1980) found that when an action was displaced from its normal temporal order in a video, almost half of the participants recalled the displaced action according to the order of the schema representation in use. Similar results have also been found with scrambled paragraphs (Kintsch, Mandel \& Kozminsky, 1977). If the story was well structured, where actions in one paragraph could be causally connected to those in another paragraph, then when the order of those paragraphs was scrambled, readers tended to mentally rearrange them into their original order, as reflected by later story recall summaries (Kintsch, Mandel \& Kozminsky, 1977). However, if the story contained no or limited causal connections between paragraphs, mentally rearranging the paragraphs was difficult as shown by poorer story summaries. Thus, recall memory can withstand scrambled paragraphs within a text. Additionally, contrary to the results of Gernsbacher (1985), another study has shown that randomizing the order of a picture story did not affect recognition memory for the left-right orientation of the images (Kraft \& Jenkins, 1977). These studies suggest that there may be no effect of scrambling well-structured picture stories for both recall and recognition memory.

\section{Predictions for Episode Component Memory}

Research on memory for episode components has failed to explain the differential memory shown across components. Recall memory has been shown to be best for the exposition and worst for the resolution (Baggett, 1979; Mandler \& Johnson, 1975). However, plan-schemas suggest that the complication and resolution should produce better memory, because these 
components contain information regarding the protagonist's goal. Conversely, Event

Segmentation Theory and the Structure Building framework both suggest that the exposition and the complication should produce better memory, because an event model or structure must be formed to make a prediction of what will happen next in the story. In the normal order, the first information to be incorporated into the event model or structure would have to be the exposition and some goal information may be required to make a prediction of future actions in the story. On the other hand, the exposition may have better memory simply because it is the first episode component presented, an advantage that would presumably be conferred to the first component in a scrambled episode. Thus, a key unanswered question is whether memory for episode components is dependent on the information that the episode components contain or instead is dependent on the order in which they are presented.

Recall memory for each episode component has been shown to be a decreasing function, where the exposition is best and the resolution is worst (Baggett, 1977; Mandler \& Johnson, 1977). However, according to plan-schemas, the complication and resolution should be best, since these episode components contain information regarding the character's goal and the attainment of the goal, respectively. The exposition would be predicted to have worse memory because it does not contain information about the character's goal, but only introduces the character and setting. From this perspective, the information presented in the exposition may be considered redundant, since setting and character information is also present during the complication and resolution.

Together Event Segmentation Theory and the Structure Building Framework predict that the exposition should have better memory than the complication or the resolution, since information in the Exposition is used to form the current event model or structure. Support for 
this hypothesis was provided by Mandler and Johnson (1977) who showed better recall for the exposition than the complication and resolution of an episode. This suggests that a new event model or structure was being constructed while processing the exposition, while subsequent information presented in the complication and resolution were mapped onto the existing event model or structure. However, research by Baggett (1979) found equivalent recall for the exposition and complication, both of which were better than the resolution. These findings suggest two distinct possibilities. First, only the exposition should have better memory, since only this information is utilized to build the current event model or structure. Conversely, the initial event model or structure may require information about the character's goal, contained in the complication, in addition to the exposition, in order to make accurate predictions about future actions that occur in the story's resolution.

Yet another more simple explanation for why memory is better for the exposition is that it is presented first, namely the primacy effect in long term memory (Tabachnik \& Joyce, 1976; Wright, Cook, Rivera, Shyan, Neiworth, \& Jitsumori, 1990). If this memory pattern was due strictly to a primacy effect, then a linear trend would be expected where the exposition is best and each subsequent component performs worse than the one preceding it. Likewise, if memory was better for the resolution then it might simply be due to a recency effect. If so, then memory should be worst for the exposition and each subsequent component should perform better than the one preceding it. The above suggests a simple serial position hypothesis: if the differential memory for episode components is simply due to their serial position (i.e., either primacy or recency effects), then changing the order in which the components are presented should change which components are remembered best and worst. Conversely, if changing the order of presentation of the components does not change which are remembered best or worst, then it 
would suggest that their differential memory is based on the information they contain, not the order in which they occur in the episode. Story grammar theories (Mandler \& Johnson, 1977; Thorndyke, 1977) would predict that memory should decrease since the components would be out of their ideal positions. However, according to Event Segmentation Theory and the Structure Building Framework, the first episode component presented should produce better memory since that information is utilized to create an event model or structure, consistent with a primacy effect but not a recency effect. Thus, these theories would predict changes to episode component memory due to their position within the episode. Conversely, plan-schemas would predict that the complication and resolution would be remembered better, regardless of their order of presentation, because of the goal/sub-goal information that is provided in them.

\section{The Representation of Events in Episode Components}

Lastly, reading times suggest that each story episode represents an event (Haberlandt, Berian, \& Sandson, 1980). However, if the character's goals are attended to in the story, then this would suggest that each episode component may represent an event. Thus, could episodes be coarse-grained events, and episode components be fine-grained events?

According to Event Segmentation Theory, memory should decrease over the course of the event. Specifically, memory should be best for the event boundary, specifically the beginning of the event, and decrease over the remainder of the event. Thus, if memory is best for the exposition and decreases monotonically for the remaining episode components, then this would suggest that the entire episode represents a coarse level event. Haberlandt, Berian, and Sandson (1980) showed that reading times are greatest for sentences around episode boundaries. This suggests that episode boundaries are used to create an event and subsequent information is mapped onto that initial structure. However, multiple studies have shown that information 
regarding the character's goals is important for comprehension and the creation of new events (Magliano \& Radvansky, 2001; Zacks \& Tversky, 2001; Lichtenstein \& Brewer, 1980). Thus, if attending to goal information is important, then it is conceivable that the information contained within each episode component represents an event. Specifically, when introduced to the characters and setting one might create an event to represent that information. The presentation of a goal and its completion, contained in the complication and resolution respectively, may themselves consist of individual events where the event model would need to be updated to represent the new information presented in the story. If this is true, then memory should be best for the first image of each episode component and decrease with each subsequent image of that component.

To test the above predictions, the current study compared both recognition and recall memory for a picture story as a function of 1) story scrambling, 2) the three episode components, and 3) the memory patterns across the individual images that compose each episode component. Specifically, the current experiment presented individual episodes from the film, The Red Balloon, in the form of a picture story. Each episode consisted of episode components in either their normal or scrambled order, leaving the images composing each episode component in their normal serial order. Participants then wrote a short summary of the episode they had viewed, followed by a recognition test, and then a self-rated comprehension measure for that episode.

\section{CHAPTER 2 - Method}

\section{Participants}

A total of 104 participants (53 females and 51 Males; Mean age $=18.79, \mathrm{SD}=1.07$ ) from Kansas State University completed the study for course credit. All participants had normal or 
corrected-to-normal vision (20/30). Two participants reported prior knowledge of the film The Red Balloon, and were removed from the analysis.

\section{Materials}

The film The Red Balloon (Lamorisse, 1956) was selected because Baggett's (1979) research identified both episodes and episode components (i.e., Exposition, Complication, and Resolution) for the film. According to her findings, the film consists of fourteen distinct episodes. A total of six episodes that showed the greatest participant agreement on episode boundaries between the film and the written narrative were selected for the current study.

A total of 144 scene images were used from the six episodes, 24 from each episode, with eight images from each episode component. Half of those images were used as target images to present the picture story, and the other half were used as distractors for the following recognition memory task. Distractors were selected that were at least one second removed from a target, but that had essentially the same "gist."

Participants' viewing positions were stabilized using a chin rest $54 \mathrm{~cm}$ from the monitor. Each image subtended $34^{\circ}$ x $27^{\circ}$ (1024 pixels x 768 pixels $)$ of visual angle. The images were presented on a 17 inch monitor (SyncMaster $957 \mathrm{MBS}$ ) at a refresh rate of $85 \mathrm{~Hz}$.

A narrative summary worksheet was created to allow participants to write a short one to two sentence summary after viewing each episode. This was followed by a 24-item picture recognition memory test. Finally, participants were asked to rate "How difficult was it to comprehend the story presented?" using a 9-point Likert scale $(1=$ Not difficult; $9=$ Very Difficult; see Appendix A).

\section{Design \& Procedure}


A 2 (scrambling [Normal vs. Scrambled]) x 3 (episode component [Exposition vs. Complication vs. Resolution]) x 4 (images per episode component) mixed design was used. The scrambling (normal vs. scrambled) factor was between-subjects, with participants randomly assigned to the two conditions. The factors of episode component and the serial order of images in each episode component were within-subject factors.

The order in which the picture story was presented was manipulated in the following ways. In the normal order condition, the picture story retained the same temporal order of the film at the episode, episode component, and image level. In the scrambled condition, both the episodes and the serial order of images within each episode component were in the same temporal order as in the film, but the order of the episode components was scrambled. Specifically, the scrambled condition eliminated the normal order (i.e., exposition, complication, and resolution) by presenting the components in one of three possible orders that had no original temporal pairings of components: 1) exposition, resolution, and complication; 2) complication, exposition, and resolution; and 3) resolution, complication, and exposition. Each of the three scrambled orders were counterbalanced and viewed twice by each participant assigned to the scrambled condition.

Participants were provided an informed consent, a visual acuity test was administered, and participants were then given instructions for the task. At the beginning of the experiment, an instruction screen was presented that indicated to the participant to press a button to begin viewing the first episode. Each scene frame was presented for two seconds, and a black screen was presented between each image for one second. After viewing an episode of the picture story, an instruction screen was presented to cue the participant to begin writing their one to two sentence summary of the episode. The recognition test was then administered, which consisted 
of 24 randomly ordered test images: the 12 target images from that episode, and 12 distractor images not seen in the picture story, but taken from nearby in the same episode. Each recognition test image was presented on the monitor until the participant made a response. If the image had been previously presented, then the participant was to press the "OLD" button, and otherwise they were to press the "NEW" button. After the recognition test, an instruction screen cued the participant to rate the difficulty of comprehending that episode. The participant then pressed the "NEXT" button to present a fixation cross before viewing the next episode in the story. This was repeated for the remainder of the story episodes. Upon completion of the experiment, participants were debriefed and thanked for their participation.

\section{CHAPTER 3 - Results}

A total of eight participants in the scrambled condition viewed a scrambled order that maintained a sequential pairing of episode components seen in the normal order (specifically, REC). In addition, two participants indicated that they had previously seen the film The Red Balloon and their data were removed from all analyses. Thus, the analysis consisted of 94 participants (52 in the normal condition and 42 in the scrambled condition).

\section{Free Recall Scoring}

Participants' summaries for each episode were coded for the content that was reported in each of the three episode components. In order to identify critical information contained within each episode component, each story episode was presented to a panel of seven raters. For each episode, the panel was presented with the twelve scene images, which composed the episode, one at a time. After presenting the images, an overview screen appeared that simultaneously presented all 12 of the scene images in temporal order from left to right, and top to bottom, identifying each episode component (as seen in Figure 1). While this overview was being 
shown, the panel wrote their summaries of the episode, and this was done for all six episodes. The panelists' summaries were compared with each other and content that was similar among the panelists was included in the content code sheets. Coded content consisted of actions taken by characters presented during that episode. An action was defined as a change in an agent's spatial position (i.e., "the boy and old woman walked into a building"), the agent's actions on another object ("the old woman released the balloon into the air"), or the agent's goal ("the boy looked for his missing balloon"). Each episode component consisted of two to three actions (see Appendix B).

Two other raters then coded participants' free recall responses for each of the six story episodes and identified actions which were included in the summary for each of the three episode components. In this way, each participant received a score for their recall completeness for each episode component. Similar to the scoring protocol of Baggett (1977), if a participant did not report any actions from an episode component, they received a score of zero. If they recalled some, but not all actions that composed the episode component, they received a score of one. If all of the actions were recalled from the episode component, they received a score of two. Thus, the possible scores for each episode summary ranged from $(0,0,0)$, recalling no actions from the three components of the story episode to $(2,2,2)$ reporting all of the actions in each component. Of the 18 scores that the raters identified per participant, their inter-rater agreement across 94 participants (for 1,692 ratings per rater) was .701 (Cohen's Kappa). The remaining disagreements were resolved by discussion between the author and the two raters. Each participant's content score was then averaged over all six story episodes, and converted into a proportion of recall, by dividing each average score by two (i.e., the maximum score a participant could receive for recalling all the actions within an episode component). The 
procedure of converting the number of actions recalled into a percentage was based on the method used by Baggett (1977).

\section{Trial Deletion}

Prior to calculating signal detection measures (sensitivity and response bias) on participants' recognition memory data, the distractor stimuli were checked to confirm that they could each be matched semantically (i.e., containing the same scene gist and background) with a target image presented in the picture story. After careful review of the distractor and target scene images, a total of ten target/distractor pairs were eliminated from the analysis for failing to meet this criterion. This resulted in an elimination of 1,880 trials (13.9\%) from a total of 13,536 trials, leaving a total of 11,656 trials remaining in the analysis.

Data trimming was done for reaction time (RT) analyses. The binned reaction time distributions for hits and correct rejections are presented in Figure 2.

[[Insert Figure 2 around here.]]

Figure 2 shows that participants' hits (i.e., correct responses to images presented during the picture story) are faster $(\mathrm{M}=1771.61, \mathrm{SD}=384.00)$ than correct rejections (i.e., correct responses to images that were not presented during the picture story $)(\mathrm{M}=1972.85, \mathrm{SD}=426.57)$ by $200 \mathrm{~ms},(t(93)=7.45, p<.001)$, suggesting that correct recognition memory RTs consist of two separate distributions. Specifically, participants were faster to decide that an image had been presented earlier than to decide that it had not been. This is consistent with much previous research showing that negative decisions take longer than affirmative decisions because negation involves a separate process (Trabasso, Rollins \& Shaughnessy, 1971). To eliminate this unwanted RT variance due to making a negative decision, only RTs for hits were further analyzed. (Another reason for not further considering the correct rejection reaction time data is 
that a total of 40 participants' data had to be removed from the overall ANOVA due to having at least one empty cell in the design for their correct rejection RTs.) Reaction time data was trimmed to eliminate the influence of outliers by using the cell mean and standard deviation of the independent variables of interest (i.e., scrambling [scrambling vs. normal order], episode component [exposition, complication, and resolution], and serial image order $\left[1^{\text {st }}, 2^{\text {nd }}, 3^{\text {rd }}\right.$, or $4^{\text {th }}$ image within a component]). Individual reaction time trials that were plus or minus three standard deviations from the cell mean were eliminated from the analysis. This resulted in the elimination of 258 trials from a total of 4,483 trials ( $5.8 \%$ of the data). Previous studies have used a two and a half standard deviation cutoff when trimming reaction times (Motes, Finlay, \& Kozhevnikov, 2006), while the proportion of data that were eliminated from the current analysis was approximately that recommended by Ratcliff (1993).

\section{Effects of Story Scrambling on Memory and Comprehension}

\section{Self-Rated Comprehension and Recall Memory}

Analyses of self-rated comprehension used a 2 (normal vs. scrambled order) x 6 (episodes) Mixed ANOVA. As predicted by story grammar theory, self-rated comprehension for the scrambled orders $(M=4.87, S D=1.30)$ was rated as more difficult than the normal order $(M$ $=4.23, S D=1.26)(F(1,92)=6.02, p=.02)$. Thus, the causal connections between episode components appeared to be an important factor in self-rated comprehension. There was a main effect of episode on self-rated comprehension $(F(5,460)=14.31, p<.001)$, showing that comprehension varied depending on the episode presented, which would be expected since each episode presented a different story. However, self-rated comprehension did not show an interaction between scrambling and episodes $(F(5,460)=1.42, p=.22)$.

[[Insert Figure 3 here.]] 
Additional evidence supporting story grammar theory was shown by recall memory for the normal versus scrambled orders. A 2 (normal vs. scrambled order) x 3 (episode components) Mixed ANOVA was used to analyze the free recall responses. Figure 3 shows the percentage of actions recalled in each of the episode components for those who viewed the story in its normal or scrambled order (see Table 1 for descriptive statistics). There was a significant main effect for scrambling $(F(1,92)=9.98, p=.002)$, where those who viewed the normal order $(M=0.44$, $S D=0.12$ ) recalled a significantly greater percentage of actions than those who viewed the scrambled orders $(M=0.36, S D=0.14)$. Thus, self-rated comprehension and recall memory support story grammar theory and its theorized importance of the causal connections between episode components.

\section{Recognition Memory}

Three separate 2 (scrambled vs. normal order) x 3 (exposition vs. complication vs. resolution) $x 4$ (serial image order $\left[1^{\text {st }}, 2^{\text {nd }}, 3^{\text {rd }}\right.$, and $\left.\left.4^{\text {th }}\right]\right)$ mixed ANOVAs were used to analyze recognition memory in terms of signal detection measures of sensitivity and bias (d' and c, respectively)(MacMillan \& Creelman, 2005), as well as recognition memory RTs.

For recognition memory, sensitivity for the normal order $(M=1.03, S D=0.34)$ was not different from the scrambled orders $(M=1.04, S D=0.32)(F(1,92)<1$, n.s. $)$. Participants were also biased to respond to scenes as "Old", and this bias did not differ between normal $(M=$ $0.32, S D=0.22)$ or scrambled orders $(M=-0.27, S D=0.20)(F(1,92)=1.18, p=.28)$. However, there was a significant main effect of scrambling on recognition memory reaction times, with significantly faster reaction times in the scrambled orders $(M=1547.32, S D=$ 270.20) than the normal order $(M=1665.76, S D=261.12)(F(1,92)=6.63, p=.03)$. Thus, participants were equally sensitive in identifying old versus new scene images in both story order 
conditions, but those who viewed the scrambled story were quicker to recognize previously viewed scenes.

The recognition sensitivity results do not support story grammar theory's claim that the presence of causal links between the episode components allowed for better memory. If fact, the recognition sensitivity results suggest that the causal links may have had a limited effect or no effect on recognition memory of the perceptual information contained in the image. However, support for story grammar theory was provided by the self-rated comprehension and recall results. These two sets of results (self-rated comprehension and recall vs. recognition memory sensitivity) suggested that there may be a difference in memory retrieval between the two story order conditions. Specifically, it appeared that conceptual information regarding the story is affected by the presence of the causal connections between episode components, as measured by the self-rated comprehension and recall scores. Conversely, perceptual information, as measured by the recognition memory sensitivity, is not affected by the presence of the causal connections in the story, though those causal connections did impact the speed of recognition retrieval. This will be described further in the discussion section.

\section{Episode Component Memory}

Recall Memory

Due to the design of the experimental task, there is no self-rated comprehension data for each episode component, because participants only rated their comprehension after viewing an entire episode.

There was a significant main effect for the episode component on recall memory $(F(2$, $184)=34.58, p<.001)($ see Figure 3$)$. In order to test whether two means were significantly different, a new F-value was calculated. To do this, first, a separate ANOVA analyzed the 
difference between the two means being compared. Then, the mean square effect term from this simplified ANOVA was divided by the mean square error term for that effect from the omnibus ANOVA. All tests with a $p$-value less than .05 were considered to be significant (See Table 7 for all pairwise comparisons). This analysis showed a significantly greater percentage of actions recalled in the exposition than the complication or resolution $(F s(1,184) \geq 12.81, p s<.001)$, and a significantly greater percentage of actions were reported in the complication than the resolution $(F(1,184)=23.64, p<.001)$.

The percentage of actions recalled for episode components interacted with scrambling the episode components $(F(2,184)=3.45, p=.034)$. The interaction was probed by calculating $F$ values for the specific means being compared (See Table 8 for all pairwise comparisons). For the normal order, there was not a significant difference between the exposition and complication $(F(1,184)=1.00, p=.32)$, but both were significantly greater than the resolution $(F s(1,184) \geq$ $12.27, p s<.001)$. In the scrambled condition, the exposition had greater recall than both the complication and resolution $(F s(1,184) \geq 15.18, p s<.001)$. In addition, the complication produced better recall than the resolution $(F(1,184)=11.45, p<.001)$. The findings for the normal order replicate the findings reported by Baggett (1979). In addition, the recall results show better recall for the exposition than the subsequent episode components, which was consistent with the predictions made by Event Segmentation Theory and the Structure Building Framework. This decreasing linear trend in the normal order was also consistent with the hypothesized primacy effect.

\section{Recognition Memory}

When both the normal and scrambled orders were averaged, there was a main effect for episode component on recognition sensitivity $(F(2,184)=11.29, p<.001)$. Figure 4 shows that 
recognition memory sensitivity was better for the complication than for the exposition $(F(1$, $184) \geq 4.76, p=.03$ )(see Table 2 for descriptive statistics and Table 5 for all pairwise comparisons). The sensitivity for the resolution was not different from the exposition or complication (Fs $(1,184) \geq 3.63, p s \geq .06)$. Response bias also differed significantly depending on the episode component $(F(2,184)=11.59, p<.001)$. Follow up $F$-tests show that the exposition had a greater "Old" response bias than the resolution $(F(1,184)=7.97, p=.006)$ (see Table 6 for all pairwise comparisons). No other comparisons were significant $(F s(1,184) \leq$ $4.29, p \geq .04)$. Recognition memory reaction time for episode components showed a nonsignificant main effect $(F(2,184)=0.29, p=.75)($ see Figure 4$)$.

[[Insert Figure 4 here.]]

The recognition memory sensitivity analyses for episode components show that it was more difficult to use the visual information contained in the exposition to discriminate between old versus new scenes than for the complication and resolution. This decrease in sensitivity may be the result of the exposition being the first episode component presented in each episode in the normal order condition. Thus, the exposition had more time to decay in memory due to its position in the episode (i.e., the recency effect). According to the recency effect hypothesis, one would expect worst performance for the episode component that was presented first and best performance for components presented last during the episode. If the linear trend for the primacy effect, as seen in the recall data, and the linear trend for the recency effect, seen in recognition sensitivity is true, then these patterns should be consistent regardless of which episode component was presented first or last in the scrambled condition. An alternative explanation is that the picture story was segmented into the story's constituent components resulting in better or worse memory for the episode component as a function of the information 
each contained (i.e., component information hypothesis). If this were true, then in the scrambled condition, better memory should be seen for the complication and resolution, regardless of when in the episode these components were presented.

\section{The Serial Position Hypothesis vs. Component Information Hypothesis}

In order to test these competing hypotheses, only data from the scrambled condition were used. Across the six episodes and across all participants in this condition, each episode component was seen in each position (i.e., first, second, and third). Recall memory, recognition memory sensitivity, and recognition reaction time data were each submitted to 3 (episode component [exposition, complication, versus resolution]) x 3 (episode component position [first, second, versus third]) within-subject factorial ANOVAs.

\section{Recall Memory}

Figure 5 shows the percentage of actions recalled in each episode component and the episode component position in the story. The data show a significant main effect for episode component $(F(2,82)=24.04, p<.001) . F$-tests showed that more actions were recalled in the exposition than both the complication and resolution $(F s(1,82) \geq 4.72$, ps $\leq .001)$ (see Table 7 for all pairwise comparisons). There was no difference in the number of actions recalled between the complication and the resolution $(F(1,82)=3.25, p=.08)$. There was not a significant effect for episode position $(F(2,82)=1.58, p=.21)$ or an interaction between episode component and position $(F(4,164)=1.07, p=.37)$, nor a significant linear trend for component position $(F(1,42)=0.01, p=.91)$.

[[Insert Figure 5 here.]]

Therefore, the results are inconsistent with the linear trend predicted by the serial position hypothesis for a primacy effect. Recall memory was not dependent on the order in which the episode components were presented. However, recall memory was dependent on the information 
that was presented in each episode component, providing support for the component information hypothesis. This is interesting because even though the episode component order was scrambled, and scrambling reduced recall, the recall pattern across episode components was similar to those viewers who saw the episode components in their normal order.

\section{Recognition Memory}

The data in Figure 6 shows the sensitivity, response bias, and reaction time results for the episode components and component position. Sensitivity showed a marginal main effect for the episode component $(F(2,82)=2.95, p=.058)$. However, there were no significant differences between the exposition, complication, and resolution on recognition memory sensitivity (Fs (1, $82) \leq 1.96, p s \geq .17)($ see Table 8 for all pairwise comparisons). There was no main effect for component position (either first, middle, or last) $(F(2,82)=0.62, p=.54)$ and no significant interaction between episode components and their position $(F(4,164)=1.12, p=.35)($ see Table 9 for descriptive statistics), nor a significant linear trend $(F(1,41)=0.97, p=.336)$. This suggests that while passively viewing the picture story, viewer's recognition memory was not dependent on when the episode component was presented. However, the marginal main effect for episode components on recognition sensitivity suggests that viewer's memory was dependent on the information presented during each episode component. This provides evidence against the linear trend predicted by the serial position hypothesis for a recency effect, which predicts worse memory for the first component presented.

[[Insert Figure 6 around here.]]

The recognition memory reaction time analyses in Figure 6 (bottom panel) show a nonsignificant main effect for episode component $(F(2,82)=0.60, p=.55)$. There was no main effect for episode component position $(F(2,82)=0.47, p=.63)$ nor an interaction between 
component and position $(F(4,164)=1.33, p=.26)($ See Table 10 for descriptive statistics $)$, nor a significant linear trend $(F(1,41)=0.91, p=.35)$. The results were inconsistent with the linear trend predicted by the serial position hypothesis of a recency effect, namely, that regardless of which episode component was presented last, recognition memory sensitivity should have been best for it. The recognition memory reaction times did not support the alternative component information hypothesis, that participant responses were based on the specific content available to them during each episode component, regardless of the order in which it was presented. This may suggest that either the retrieval of perceptual information does not vary between episode components regardless of when it was presented, or that the reaction time measure was not sufficiently sensitive to differentiate recognition of perceptual information contained within episode components.

\section{The Representation of Events in Episode Components}

Previous research has suggested that the story episode represents an event based on reading times for episode components of a story (Haberlandt, Berian, Sandson, 1980). Nevertheless, there is still the question of whether our story episodes represent events as proposed in Event Segmentation Theory. According to Event Segmentation Theory, the memory pattern for an event should consist of better memory at the beginning of the event and worse memory later on in the event. The recall data for the normal order suggest that the story episode does represent an event, however the recognition memory results would suggest the opposite. A possible explanation for these opposing effects for recall and recognition is in terms of Mandler's dual-process theory of recognition memory (Mandler, 2008). It may be that the conceptual memory is represented as an event, although the perceptual memory was lost by the time it was tested in the recognition memory task. If this is true, one would expect that testing recognition 
memory immediately after viewing each episode component would provide evidence from recognition memory of episodes being representative of events. However, the current recall and recognition results are inconclusive as to whether the episode is representative of an event. A further possibility is that the participants were in fact segmenting the story into events, but that their events were represented at the level of each episode component.

Neither self-rated comprehension nor recall could be measured at the sub-component level of analysis. Self-comprehension measures were only collected after viewing an entire episode, and recall data could only be coded at the episode and component level.

\section{Recognition Memory}

The serial image order that composed each episode component was analyzed to determine if there were distinctive recognition memory patterns consistent with Event Segmentation Theory. Specifically, the question was whether there was a main effect in recognition memory in which sensitivity is best at the beginning of the component and worst at the end. If so, reaction times should be fastest at the beginning of the component and slowest at the end. Figure 7 presents sensitivity, bias, and RTs for the serial image order averaged across the normal versus scrambled conditions, since there were no interactions involving scrambling and component image serial order for sensitivity $(F(3,276)=0.41, p=.73)$, bias $(F(3,276)=$ $0.08, p=.97)$, or RT $(F(3,276)=2.01, p=.11)$.

\section{[[Insert Figure 7 about here]]}

Results show that there was a main effect of serial image order for sensitivity $(F(3,276)$ $=11.29, p<.001)$, bias $(F(3,276)=11.59, p<.001)$, and reaction time $(F(3,276)=9.76, p<$ .001). However, each of these main effects was qualified by significant serial image order by episode component interaction. Thus, the pattern of results for the serial image order effect was 
not the same for each episode component, where certain episode components may in fact be representative of an event. The interaction between scrambling and episode component was not significant for sensitivity $(F(2,184)=0.17, p=.85)$, bias $(F(2,184)=1.15, p=.32)$, or reaction time $(F(2,184)=1.25, p=.29)$. Additionally, the scrambling $\mathrm{x}$ episode component $\mathrm{x}$ serial image order interaction was not significant for sensitivity $(F(6,552)=1.77, p=.10)$, bias $(F(6,552)=1.15, p=.33)$, or reaction time $(F(6,552)=0.36, p=.91)$.

[[Insert Figure 8 about here]]

Figure 8 presents sensitivity, bias, and reaction times for the serial image order separated by episode components. Sensitivity significantly differs according to the specific episode components $(F(6,552)=21.61, p<.001)($ See Table 11 for descriptive statistics). The complication and resolution produced a relatively consistent pattern. The strongest serial image order sensitivity effect is for the complication, which shows a u-shaped function. F-tests were used to compare serial image positions (i.e., image 1 vs. 2, 2 vs. 3, and 3 vs. 4)(see Table 12 for all comparisons). Sensitivity for the first image of the complication was greater than for the second image $(F(1,552)=7.86, p=.005)$, while the fourth image had significantly greater sensitivity than the third image $(F(1,552)=32.48, p<.001)$. Sensitivity in the resolution was greater in the fourth image than the third image $(F(1,552)=28.38, p<.001)$, however no other significant differences were observed $(F s(1,552) \leq 7.42$, ps $\geq .007)$. The sensitivity pattern seen in the resolution was relatively consistent with the pattern in the complication, however sensitivity was not greater for the first image than the second image. In the exposition, sensitivity was significantly worse in the first image than the second image $(F(1,552)=90.38, p$ $<.001)$, and no other significant differences were present in the exposition $(F(1,552) \leq 0.36, p$ $\geq .55)$. 
The u-shaped pattern seen for sensitivity in the complication (see Figure 8) appears on the surface to be representative of a primacy and recency effect. However, this greater sensitivity was not due to the images being the first and last images in the entire picture story episode. These images were actually the fifth and eighth images in the serial image sequence for the episode (in the normally ordered condition). This increase in recognition sensitivity for the beginning and ending of the complication suggests that this episode component may be represented as an event, as suggested by Event Segmentation Theory. Recognition sensitivity was also better for the end of the complication, which is not predicted by Event Segmentation Theory. Therefore, the complication itself does not appear to be composed of a single event, although it may be represented by two events, with the first event beginning with the first image of the complication, and the second event beginning with the last image of the complication. In addition, memory patterns representative of an event are not found for the exposition or resolution. In sum, it may be that each episode component was not represented in recognition memory as an event. While the data suggests that event-like structures may be present, this is still speculative and more evidence is needed to determine where any potential events may be in the episode components.

Reaction times for image serial position within episode components also differed depending on the episode component $(F(6,552)=2.78, p=.01)($ see Table 13 for descriptive statistics and Table 14 for all statistical comparisons). A similar pattern for reaction time was seen again for the exposition and complication, where RT tends to be slower for the first image and becomes progressively quicker for subsequent images in that component. F-tests were used to probe the interaction. Sequential images were compared within each episode component for a total of 12 paired comparisons (i.e., image 1 vs. 2, 2 vs. 3, 3 vs. 4, and 1 vs. 4 per episode 
component). The second and fourth image in the exposition produced significantly faster reaction times compared to the first image $(F s(1,552) \geq 10.00, p s \leq .002)$. The last image in the complication produced significantly faster reaction times compared to the first $(F(1,552)=$ $20.29, p<.001)$ and marginally faster reaction times to the third image $(F(1,552)=8.12, p=$ .0045). All other comparisons produced non-significant effects $(F s(1,552) \leq 5.13, p s \geq .02)$. If each episode component was representative of an event, recognition memory reaction times would have been expected to increase over the images composing the episode component. Specifically, recognition memory reaction times would be fastest for the first image and slowest for the last image in the component. The reaction times for the episode components are in fact opposite to this prediction. Therefore, each episode component does not appear to be representative of an individual event, as defined by Event Segmentation Theory.

An alternative explanation for why longer reaction times are found at the beginning of the exposition and complication may be that the information presented in these images has been converted from perceptual to conceptual information. This is supported by better recognition sensitivity for the remaining images in the exposition (images $2-4$ ), which have yet to be converted into conceptual information, thus these images tended to have faster reaction times. A relatively similar pattern was found for the complication, however the first image has a slower reaction time and also better recognition memory. This trend seemed to be present in the resolution, where the last image has better recognition memory sensitivity and shorter reaction times. However, this perceptual conversion explanation is speculative regarding the interpretation of the data at the image level, and future research should examine the time course of perceptual and conceptual information processing for picture stories. 


\section{Chapter 4 - Discussion}

According to a number of story grammar theories (Kintsch, 1977; Mandler \& Johnson, 1977; Thorndyke, 1977; van Dijk, 1977), a story can be decomposed into distinct episodes, which can be further broken down into three distinct episode components. These episode components are arranged in a specified order, with causal connections linking one component to the next. When this causal linkage between components is replaced by another connection (e.g., temporal), then memory and comprehension for the episode should suffer. Previous research has shown mixed results as to whether scrambling a story results in worse memory, with some studies showing worse memory (Gernsbacher, 1985; Thorndyke, 1977), and some showing no effect of scrambling on memory (Kintsch, Mandel \& Kozminsky, 1977; Kraft \& Jenkins, 1977). However, an alternative prediction has suggested that memory might be better after eliminating the links between episode components by scrambling the component order. According to Event Segmentation Theory (Zacks, Speer, Sallow, Braver, \& Reynolds, 2007), information is broken into discrete chunks which are referred to as events. When processing a new event, cognitive processing increases for the beginning of the event and then decreases thereafter. This increased processing results in better memory for event boundaries (Schwan \& Garsoffky, 2004; Swallow, Zacks, \& Abrams, 2009). Thus, by eliminating the causal links between episode components due to scrambling them, viewers might perceive a greater number of events

In addition, consistent memory patterns have been found for each of the episode components, where the exposition is best and the resolution is worst (Baggett, 1979; Mandler \& Johnson, 1977). However, no explanation has been given for this pattern for episode components. The present study predicted, based on Event Segmentation Theory and the Structure Building Framework that memory for the exposition and complication may be best 
because the information presented at the beginning of an episode is used to create a new event model or structure to predict what will occur next. On the other hand, this pattern of results is also consistent with a linear trend predicted by the serial position curve's primacy effect. Conversely, plan-schemas may be used to recall information about the goals in the story. Therefore, the current study alternatively predicted that the complication and resolution may be best since the complication presents the goal and the resolution shows the character achieving the goal. Interestingly, such a pattern would also be consistent with a linear trend representing a recency effect. Thus, if these serial position hypotheses are correct, then these linear trends should be present regardless of the order of the episode components. Conversely, if memory differs between episode components, regardless of there order, then memory is dependent on the information that the components contain.

Finally, research has shown that cognitive processing increases at the beginning of a new story episode (Haberlandt, Berian \& Sandson, 1980), which is consistent with creating a new event according to Event Segmentation Theory. This increased cognitive processing is associated with better memory for the beginning of an event than its remainder. This suggests the possibility that each episode could be composed of three smaller events represented by each of the episode components.

The present study found support for story grammar theories with participants rating the story episodes as more difficult to understand and a decrease in the percentage of actions recalled for the scrambled episodes. The decrease in self-reported comprehension and recall is consistent with the elimination of the causal links between episode components. However, Event Segmentation Theory (Zacks, Tversky, \& Iyers, 2001) may explain the data by suggesting that the effect of scrambling the episode components produced a much larger memory decrement 
than the memory improvement due to perceiving numerous small events. A further alternative may be that the boundaries between episode components were not actual event boundaries, but were instead points within events. If so, then scrambling at non-event boundaries would be highly disruptive for memory (Boltz, 1992; Schwan, Garsoffky, \& Hesse, 2000). However, this seems to be unlikely since it would be inconsistent with the claim that events are segmented at conceptual changes, such as changes to character goals and causal relationships. These factors, along with changes in temporal and spatial relationships of the characters in the story, would make segmenting more likely at the episode component boundaries, especially in the scrambled condition.

Some of the memory measures were unaffected by scrambling, specifically recognition memory sensitivity and bias. This is consistent with the results of Kraft and Jenkins (1977) who showed no effect of picture story scrambling on recognition memory for left vs. right picture orientation. Similarly, Brewer and Dupree (1983) found no effect of scrambling on recognition memory for information conforming to a plan-schema, but a large effect for recall. Nevertheless, a surprising finding in the current study shows a difference in recognition memory reaction time between story orders, such that the scrambled condition had a faster reaction time than the normal order. While this could be taken as evidence supporting the hypothesis based on Event Segmentation Theory that scrambling could improve memory, it may instead be explained in terms of participants in the scrambled condition using a different memory processes to make their responses. Specifically, in the scrambled condition, perceptual information may have been maintained in working memory for a longer period of time in order to try and form a better conceptual representation of what had happened in the story, thus producing faster recognition RTs. Conversely, in the normal order, the perceptual information may have been transformed to 
conceptual information faster than in the scrambled condition, because it fit better with a standard story grammar schema, making easier to encode and store as conceptual information, producing better recall.

Mandler's (2008) dual-process theory of recognition memory can help explain our results because it describes recognition processes occurring through two parallel routes. The first route is based on familiarity of the perceptual information presented at test compared with the structure represented in memory. These memory representations decay over time, but after a recent presentation or repeated presentations, the memory representation may become stronger. The second route consists of recollection of conceptual information. These memory representations are semantically organized, which allows for search and retrieval of information contained within the representation. This memory search process requires more time, resulting in longer reaction times. Taking these two routes under consideration, it may be that those who viewed the scrambled story were basing their judgments on familiarity, namely matching the scene image to a perceptually similar representation in memory.

In the current experiment, such a familiarity-based process in long-term memory could have been used quite effectively due to the relatively short retention interval between the episode presentation and the start of the recognition test, which was 73 seconds on average. For those in the normal order condition, their structural representation of the story was likely elaborated due to the semantic organization of the story, making it a more complex mental representation. Responses would then require the use of recollective processes involving more complex yet semantically organized story memory representations, resulting in longer reaction times. The free recall responses confirm that those viewing the scrambled order made their responses 
without a strong semantically organized representation of the story, as shown by the lower recall scores for this condition, whereas the opposite was true of those who saw the normal order.

$$
\text { [[Insert Figure } 9 \text { about here.]] }
$$

The processing time needed for these two routes of recognition can be represented with a hazard function, shown in Figure 9. The hazard function represents the probability of the participant making a hit on the recognition task, at each point in time. The hazard function is calculated by dividing the relative frequency of reaction times for an RT bin by the relative frequency of the remaining RTs following it, with bins being given the center value of their range. These hazard values were then smoothed by averaging the value of the current bin (which was double weighted) together with the values of the immediately preceding and following bins (which were single weighted). The magnitude of the difference between the scrambled and normal conditions is also shown in Figure 9.

Based on the logic of Mandler's Dual Process Theory, those who viewed the normal story should have taken more time to reach a decision, whereas those in the scrambled condition should have taken less time to do so. Specifically, the hazard function for the normal story condition should have a shallower slope then the scrambled story condition. As shown in Figure 9 , the slope for the scrambled condition is indeed steeper than the normal story condition, consistent with the hypothesis that those in the normal story condition had a more complex representation of the picture story than those in the scrambled condition, and thus, required more time to search their memory representation of the story. Conversely, those in the scrambled story assumedly produced less elaborate story representations, which were more perceptually based, thus searching their memory for the recognition task required less time. Interestingly, these differences appear to have gradually increased from early RTs (at roughly $750 \mathrm{~ms}$ ) until 
around $1,150 \mathrm{~ms}$, and thereafter declined or remained steady. Thus, beyond the very earliest responses, for several hundred milliseconds, responses became increasingly faster in the scrambled condition, and only after approximately 1,150 ms post-stimulus did recognition responses become roughly equally probable in the two conditions.

Let us now consider the results for memory as a function of episode component. The results found for recall were consistent with predictions based on Event Segmentation Theory and the Structure Building Framework, with memory for the exposition being best, when a new event model or structure would be created. However, such predictions are not supported by the recognition memory results, which showed the opposite trend. Interestingly, these opposing results found for recall and recognition are consistent with our discussion of Mandler's dualprocess model. Specifically, worse recognition sensitivity for the exposition would occur as time passed during the retention interval and surface level visual details were transformed to more conceptual information in long-term memory (Brewer \& Dupree, 1983; Gernsbacher, 1985). Thus, the information retained from the exposition, which showed superior recall would be such conceptual information. Conversely, the complication and resolution had better recognition memory because they had not yet been converted into conceptual memory representations, as shown by worse recall memory. Thus, the pattern of results across recognition sensitivity and free recall would be explained in terms of decreasing familiarity over time (i.e., perceptual information loss) coupled with increasing recollective processes (i.e., conceptual information).

It should be pointed out, however, that these opposing patterns found for recall and recognition were not due to simple primacy or recency effects, respectively. When the episode components were scrambled, there was no decreasing or increasing linear trends as a function of 
component order were present. Instead, differences were still found between the episode components, regardless of their serial position in the picture story.

One might claim that these results provide evidence against the argument based on perceptual information loss. Specifically in the scrambled order, those episode components that were presented first did not show worse recognition memory than subsequent episode components. Instead, the scrambled story condition shows evidence that performance for episode components is a result of the information provided regardless of when in the story it is presented. Thus, our explanation of the opposing results for recognition and recall across components in terms of Mandler's dual-process theory cannot make use of elapsed time, at least not in units roughly corresponding to the three episode components.

Recognition memory within episode components did not support the hypothesis that episode components are events, as defined by Event Segmentation. Specifically, there was little evidence of recognition memory decreasing from the first image in each component to the last image. Though the present data do not support this hypothesis, this conclusion must be qualified. Clearly, each episode does contain actions by characters, thus there should be one or more events in each episode, and likely in each component. However, the current recognition sensitivity data does not support the hypothesis that event boundaries coincide with episode component boundaries.

\section{Limitations}

The current study has several limitations. First, viewers were shown only four images per episode component. This is a limitation because the duration of each component varied in the film. This variance made it difficult to select scene images that best represented each episode component. For example, when the viewer was presented with the next image in the picture 
story, it may have been an image that would have been seen two seconds later to as much as 93 seconds later, in the film. This temporal difference between two images may have resulted in the loss of some conceptual information in the picture story. For instance, it may become more difficult to interpret actions or character intentions, or to comprehend how two images are related when the information in the setting differs. In addition, only one story episode was tested at a time. Therefore it is unknown if the current findings are representative of those who have viewed an entire picture story.

Another limitation of the current study is that the picture story came from only one film. The data would clearly be more generalizable if multiple different films were used. However, given the amount of time required to design such a study for only a single film, doing so for multiple films in a single study would be prohibitive. Nevertheless, each episode in the picture story does represent different situations which, in turn, increase the generalizability of the current findings.

Finally, the current study did not have participants segment the picture story into separate events. Therefore, it is unknown whether or not the participants in the scrambled condition perceived more events than those in the normal condition. It may have been that those in the scrambled condition perceived an equivalent number of events to those in the normal condition, which may be one reason why there was no effect of scrambling on recognition memory. Scrambling has been suggested to increase the number of perceived events in a story, however with the current data it is unclear as to whether scrambling episode components actually produced an increase in the number of perceived events. In addition, the current hypothesis that episode components were composed of events was tested indirectly using recognition memory data for event boundaries. However, if event segmentation data for the picture story had been 
collected, then a stronger test could have been conducted to determine if participants' event boundaries do coincide with episode components.

\section{Future Directions}

As noted above, it is unclear whether viewers in the scrambled condition perceived more fine-grained events than those in the normal condition. This is important since previous research has shown that those instructed to parse a video into fine-grained units recalled more than those instructed to parse the video into coarse-grained units (Lassiter, 1988; Lassiter, Stone, \& Rogers, 1988). Future research should replicate the present design while instructing viewers to segment the picture story into events. By segmenting the picture story, we can explicitly test whether episode and episode component boundaries map onto event boundaries. In addition, viewers should be given instructions to either segment the picture story into its largest meaningful unit (i.e., coarse-grained events) or into its smallest meaningful units (i.e., fine-grained units). Such instructions should maximize the cognitive processing differences between these two groups. Thus, if the order of the episode components is important for memory, then scrambling the components should produce a decrement in memory, independent of grain of event

segmentation. However, if the frequency of perceived events is more important than component order, then fine-grained events should produce better memory than coarse-grained events, independent of story order. The results of recent research that had viewers segment "The Red Balloon" into events (Zacks, Speer, \& Reynolds 2009) could be used to directly determine the relationship between episodes, episode components, and events in picture stories, and more generally in movies.

Scrambling picture stories at the episode component level has an effect on comprehension and recall, however it is unclear if these effects would generalize to scrambling 
at the episode level. Previous films like Pulp Fiction (Bender \& Tarantino, 1994) and Memento (Todd, Todd, \& Nolan, 2000) have scrambled the story timeline at a higher level than episode components, however it is an open question as to whether this form of scrambling would produce a decrement in self-rated comprehension and recall for the story. One may hypothesize that due to the elimination of the temporal order at the episode level that this would remove cues between story episodes, which would result in a decrease in comprehension and recall memory. However, it is also possible that scrambled story episodes can be reordered into their normal temporal order, which would result in no effect of scrambling at the episode level.

Research on event processing during a picture story is relevant for research on the time course of scene processing. Our data suggests that visual information processing differed depending on the specific image within the episode component being presented. Specifically, processing of the first image in the complication differed from the second and third image. This happens to be consistent with changes in cognitive processing for event boundary images compared to non-event boundary images (Schwan \& Garsoffky, 2004; Swallow, Zacks, \& Abrams, 2009). This is also similar to research showing that visual processing of a single scene also varies over time, as shown by eye-movement measures and visual search tasks (Pannasch, Schulz, \& Velichkovsky, 2010; Unema, Pannasch, Joos, \& Velichkovsky, 2005; Malcolm \& Henderson, 2010). However event processing may help in identifying when this processing would differ and what information is being extracted during different time points (Fei-Fei, Iyer, Koch, \& Perona, 2007). The distribution of attention varies over the time course of a single eye fixation on a single scene (Larson, Loschky, Ringer \& Kridner, 2010), and over a sequence of multiple fixations on a single scene (Pannasch, Schulz, \& Velichkovsky, 2010; Unema, Pannasch, Joos, \& Velichkovsky, 2005), as well as over multiple scenes (Swallow \& Jiang, 
2010). The effects of eye movements, attention, and processing time on single scenes should be examined to determine their generalizability to the perception of events in picture stories and videos. Specifically, how does attention vary over different time-scales of event segmentation in complex events? How do eye movements vary over the course of a complex event, and are there typical patterns observed of the event? What is the time course of event identification? Specifically, how is information regarding scene gist used to comprehend complex events in picture stories and movies?

The results of the current study may not be completely generalizable to cognitive processing of television, since participants viewed one episode at a time, whereas multiple episodes may be presented during a television program. However, some general claims can be proposed regarding television processing assuming that a television episode is representative of a story episode according to story grammar theories.

Scrambling a television program at the level of the episode components would be predicted to hurt the viewer's comprehension and recall of the program, because the causal links between the episode components would have been eliminated. However, worse recall and comprehension would not be a result of an inability to extract sufficient perceptual information from the story since, recognition memory sensitivity would be equivalent between a normal and temporally scrambled story. Therefore, scrambling the episode components of a television program would not be detrimental to our lower-level perceptual abilities, but would be detrimental to higher-level cognitive processes. These processes would include the organization of the perceptual details of the story that was seen and transforming it into a conceptual code of actions or other semantic code. Evidence of such higher-level cognitive effects was observed in the current thesis by decomposing the episodes into their separable components. For instance, in 
the current study, recall was best in the exposition and worst in the resolution. Generally speaking, this general decrease in recall over story components was similar in both the scrambled and unscrambled conditions. This suggests that the trend observed for memory of conceptual information from episode components was dependent on the information that was provided in each episode component, and not dependent on the temporal relationships of those episode components. Therefore, if similar processes occur in comprehending and remembering television programs, and if those television episode components are scrambled, one would hypothesize that recall memory would be dependent on the information contained within each episode component and not dependent on their temporal relationship with one another.

Overall, this study showed that scrambling the temporal order of episode components resulted in a decrement in story comprehension and memory for recalled actions. Interestingly, although scrambling the picture story did not affect sensitivity to recognition memory for perceptual information, it did produce faster recognition reaction times for the scrambled story. When examining memory for episode components, it was found that recall was best for the exposition and recognition memory was best for the resolution. Interestingly, these effects found for recall and recognition memory could not be explained by temporal factors, specifically a primacy or recency effect respectively. Instead, recall was best for the exposition regardless of when it was presented during the picture story. Likewise, recognition memory seemed to be better for specific episode components regardless of when they were presented in the picture story. These interesting findings suggest that people were sensitive to certain information contained within each episode component; in addition, they were sensitive to that information regardless of when the components were presented. This may have been the result of implicitly segmenting the picture story into its separate episode components, and, if so, it implies that 
viewers were attending to particular features within the picture story that cued them to the episode components' functional roles in the episode structure.

The current recall and recognition sensitivity results for each episode component may be explained by the features described by the Event Indexing Model (Zwaan \& Radvansky, 1998). This model identifies five features ("time, space, causation, intentionality, and protagonist" p. 167) that are attended to during text comprehension. Specifically, while reading a sentence in a story the reader creates a situational model for that sentence. If the temporal features in the subsequent sentence remain the same (i.e., there is temporal contiguity), then the information in the second sentence is easily added to the situation model. However, if the next phrase begins with, "One week later," then the temporal feature needs to be updated, which results in an increase in the processing load for that story. Additionally, if more than one feature needs to be updated, then there should be a greater increase in processing demands to update these story features.

Each image in our picture story could be coded regarding the features identified by the Event Indexing Model. A comparison could then be made between the number of features that are changing in each episode component and viewers' recall and recognition for each episode component. One hypothesis would predict that better recall memory for an episode component would be the result of having fewer changes to conceptual features (i.e., causality and intentionality) during that component, and worse recall memory if more conceptual features changed during that episode component. A similar set of predictions could be made for recognition memory regarding the number of changes of perceptual features (i.e., spatial, temporal, and protagonist). If these hypotheses were supported, then it would suggest that the 
differences in memory for episode components may be the result of specific features, either conceptual or perceptual, that are attended to while processing the picture story. 


\section{References}

Baggett, P. (1979). Structurally equivalent stories in movie and text and the effect of the medium on recall. Journal of Verbal Learning and Verbal Behavior, 18, 333 - 356.

Bender, L. (Producer), \& Tarantino, Q. (Writer/Director). (1994). Pulp Fiction [Motion picture]. United States: Miramax Films.

Brewer, W. F., \& Dupree, D. A. (1983). Use of plan schemata in the recall and recognition of goal-directed actions. Journal of Experimental Psychology: Learning, Memory, and Cognition, 9(1), 117 -129.

Boltz, M. (1992). Temporal accent structure and the remembering of filmed narratives. Journal of Experimental Psychology: Human Perception and Performance, 18(1), 90 - 105.

Carroll, J. M. \& Bever, T. G. (1975). Segmentation in cinema perception. Science, 191, 1053 1055.

Fei-Fei, L., Iyer, A., Koch, C. \& Perona, P. (2007). What do we perceive in a glance of a realworld scene? Journal of Vision, 7(1):10, 1 - 29.

Gernsbacher, M. A. (1983). Memory for the orientation of pictures in nonverbal stories: Parallels and insight into language processing. Unpublished doctoral dissertation, University of Texas at Austin, Austin.

Gernsbacher, M. A. (1985). Surface information loss in comprehension. Cognitive Psychology, $17,324-363$

Gernsbacher, M. A. (1990). Language comprehension as structure building. Hillsdale, New Jersey: Lawrence Erlbaum Associates, Inc.

Gernsbacher, M. A. (1997). Two decades of structure building. Discourse Processes, 23(3), 265 $-304$. 
Gernsbacher, M. A., Varner, K. R., \& Faust, M. E. (1990). Investigating differences in general comprehension skill. Journal of Experimental Psychology: Learning, Memory, and Cognition, 16(3), $430-445$.

Haberlandt, K. (1984). Story grammar and reading time of story constituents. Poetics, 9, 99 118.

Haberlandt, K., Berian, C., \& Sandson, J. (1980). The episode schema in story processing. Journal of Verbal Learning and Verbal Behavior, 19, 635 -650.

Hanson, C. \& Hirst, W. (1989). On the representation of events: A study of orientation, recall, and recognition. Journal of Experimental Psychology: General, 118(2), 136 - 147.

Hard, B. M., Tversky, B., \& Lang, D. S. (2006). Making sense of abstract events: Building event schemas. Memory \& Cognition, 34(6), 1221 - 1235.

Kintsch, W. (1977). On comprehending stories. In M. A. Just \& P. A. Carpenter (Eds.), Cognitive Processes in Comprehension (pp. ). Hillsdale, New Jersey: Erlbaum.

Kintsch, W., Mandel, T. S., \& Kozminsky, E. (1977). Summarizing scrambled stories. Memory \& Cognition, 5(5), $547-552$.

Kraft, R. N. \& Jenkins, J. J. (1977). Memory for lateral orientation of slides in picture stories. Memory \& Cognition, 5(4), 397 - 403.

Kurby, C. A., \& Zacks, J. M. (2007). Segmentation in the perception and memory of events. Trends in Cognitive Science, 12(2), 72 - 79.

Lamorisee, A., (Writer/Director). (1956). The Red Balloon [motion picture] France: Films Montsouris.

Larson, A.M., Loschky, L.C., Ringer, R., \& Kridner, C. Attention modulates gist performance between central and peripheral vision. Poster presented at the $10^{\text {th }}$ Annual Meeting of the Vision Sciences Society, Naples, FL. 
Lassiter, G. D. (1988). Behavior perception, affect, and memory. Social Cognition, 6(2), 150 176.

Lassiter, G. D., Stone, J. I., \& Rogers, S. L. (1988). Memorial consequences of variation in behavior perception. Journal of Experimental Social Psychology, 24, 222 - 239.

Lichtenstein, E. H., \& Brewer, W. F. (1980). Memory for goal-directed events. Cognitive Psychology, 12, $412-445$.

Macmillan, N. A., \& Creelman, C. D. (2005). Detection Theory: A User's Guide (2nd ed.). Mahwah, N.J. : Lawrence Erlbaum Associates.

Magliano, J. P., Miller, J., \& Zwaan, R. A. (2001). Indexing space and time in film understanding. Applied Cognitive Psychology, 15, 533 - 545.

Magliano, J. P., \& Radvansky, G. A. (2001). Goal coordination in narrative comprehension. Psychonomic Bulletin \& Review, 8(2), 372 - 376.

Malcolm, G. L. \& Henderson, J. M. (2010). Combining top-down processes to guide eye movements during real-world scene search. Journal of Vision, 10(2):4, $1-11$.

Mandler, G. (2008). Familiarity breeds attempts: A critical review of dual-process theories of recognition. Perspectives on Psychological Science, 3(5), 390 - 399.

Mandler, J. M. \& Goodman, M. S. (1982). On the psychological validity of story structures. Journal of Verbal Learning \& Verbal Behavior, 21(5), 507 - 523.

Mandler, J. M., \& Johnson, N. S. (1977). Remembrance of things parsed: Story structure and recall. Cognitive Psychology, 9, $111-151$.

Motes, M. A., Finlay, C. A. \& Kozhevnikov, M. (2006). Scene recognition following locomotion around a scene. Perception, 35(11), $1507-1520$. 
Newston, D. (1973). Attribution and the unit of perception of ongoing behavior. Journal of Personality and Social Psychology, 28(1), 28 - 38.

Newston, D., \& Engquist, G. (1976). The perceptual organization of ongoing behavior. Journal of Experimental Social Psychology, 12, 436 - 450.

Pannasch, S., Schulz, J., \& Velichkovsky, B. (2010). Explaining visual fixation durations in scene perception: Are there indeed two distinct groups of fixations? Poster presented at the $10^{\text {th }}$ Annual Meeting of the Vision Sciences Society, Naples, FL.

Ratcliff, R. (1993). Methods for dealing with reaction time outliers. Psychological Bulletin, 114(3), $510-532$.

Reynolds, J. R., Zacks, J. M., \& Braver, T. S. (2007). A computational model of event segmentation from perceptual prediction. Cognitive Science, 31, $613-643$.

Schwan, S., \& Garsoffky, B. (2004). The cognitive representation of filmic event summaries. Applied Cognitive Psychology, 18, 37 - 55.

Schwan, S., Garsoffky, B., \& Hesse, F. W. (2000). Do film cuts facilitate the perceptual and cognitive organization of activity sequences? Memory \& Cognition, 28(2), 214 - 223.

Speer, N. K., \& Zacks, J. M. (2005). Temporal changes as event boundaries: Processing and memory consequences of narrative time shifts. Journal of Memory and Language, 53, $125-140$.

Speer, N. K., Zacks, J. M., \& Reynolds, J. R. (2007). Human brain activity time-locked to narrative event boundaries. Psychological Science, 10(5), $449-455$.

Swallow, K. M., \& Jiang, Y. V. (2010). The attentional boost effect: Transient increases in attention to one task enhance performance in a second task. Cognition, 115(1), $118-132$. 
Swallow, K. M., Makovski, T., \& Jiang, Y. V. (May, 2009). The attentional boost effect. Poster session presented at the annual meeting of the Visual Sciences Society, Naples, FL.

Swallow, K. M., Zacks, J. M., \& Abrams, R. A. (2009). Event boundaries in perception affect memory encoding and updating. Journal of Experimental Psychology: General, 138(2), $236-257$.

Tabachnik, B., \& Brotsky, S. J. (1976). Free recall and complexity of pictorial stimuli. Memory and Cognition, 4(5), $466-470$.

Thorndyke, P. W. (1977). Cognitive structures in comprehension and memory of narrative discourse. Cognitive Psychology, 9, 77 - 110.

Todd, J. (Producer), Todd, S. (Producer), \& Nolan, C (Director). (2000). Memento [Motion picture]. Newmarket Capital Group.

Trabasso, T., Rollins, H., \& Shaughnessy, E. (1971). Storage and verification stages in processing concepts. Cognitive Psychology. 2(3), 239 - 289.

Unema, P. J., Pannasch, S., Joos, M., \& Velichkovsky, B. M. (2005). Time course of information processing during scene perception: The relationship between saccade amplitude and fixation duration. Visual Cognition, 12(3), 473 - 494.

van Dijk, T. A. (1977). Semantic macro-structures and knowledge frames in discourse comprehension. In M. A. Just \& P. A. Carpenter (Eds.), Cognitive Processes in Comprehension (pp. 3 - 32). Hillsdale, New Jersey: Erlbaum.

Wright, A. A., Cook, R. G., Rivera, J. J., Shyan, M. R., Neiworth, J. J., \& Jitsumori, M. (1990). Naming, rehearsal, and interstimulus interval effects in memory processing. Journal of Experimental Psychology: Learning, Memory, and Cognition, 16(6), 1043 - 1059. 
Zacks, J. M., Braver, T. S., Sheridan, M. A., Donaldson, D. I., Snyder, A. Z., Ollinger, J. M., et al. (2001). Human brain activity time-locked to perceptual event boundaries. Nature Neuroscience, 4(6), $651-655$.

Zacks, J. M., Kumar, S., Abrams, R. A., \& Mehta, R. (2009). Using movement and intentions to understand human activity. Cognition, 112, 201-216.

Zacks, J. M., Speer, N. K., Reynolds, J. R. (2009). Segmentation in reading and film comprehension. Journal of Experimental Psychology: General, 138(2), 307 - 327.

Zacks, J. M., Speer, N. K., Swallow, K. M., Braver, T. S., \& Reynolds, J. R. (2007). Event Perception: A mind-brain perspective. Psychological Bulletin, 133(2), 273 - 293.

Zacks, J. M., \& Tversky, B. (2001). Event structure in perception and conception. Psychological Bulletin, 127, 3-21.

Zacks, J. M., Tversky, B., \& Iyer, G. (2001). Perceiving, remembering, and communicating structure in events. Journal of Experimental Psychology: General, 130(1), 29 - 58.

Zwaan, R. A., \& Radvansky, G. A. (1998). Situation models in language comprehension and memory. Psychological Bulletin, 123(2), 162 - 185. 


\section{Appendix A}

Narrative 1

How difficult was it to comprehend the story presented?

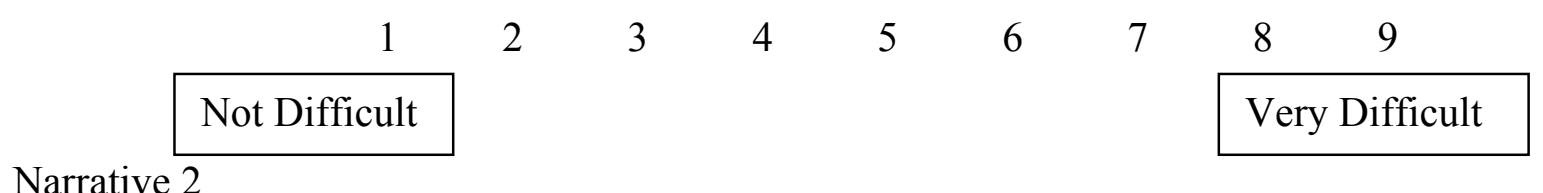

How difficult was it to comprehend the story presented?

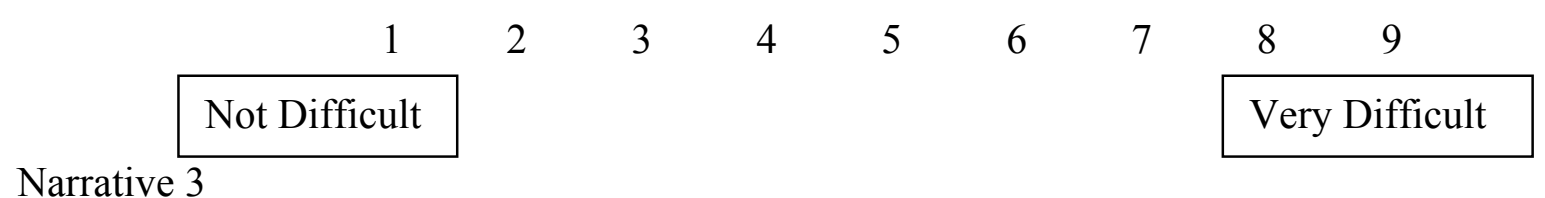

How difficult was it to comprehend the story presented?

\begin{tabular}{|c|c|c|c|c|c|c|c|c|}
\hline 1 & 2 & 3 & 4 & 5 & 6 & 7 & 8 & 9 \\
\hline Not Difficult & & & & & & & & Difficult \\
\hline
\end{tabular}


Narrative 4

How difficult was it to comprehend the story presented?

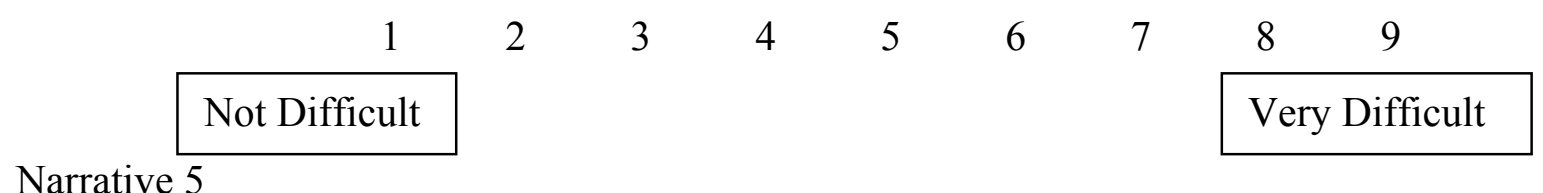

How difficult was it to comprehend the story presented?

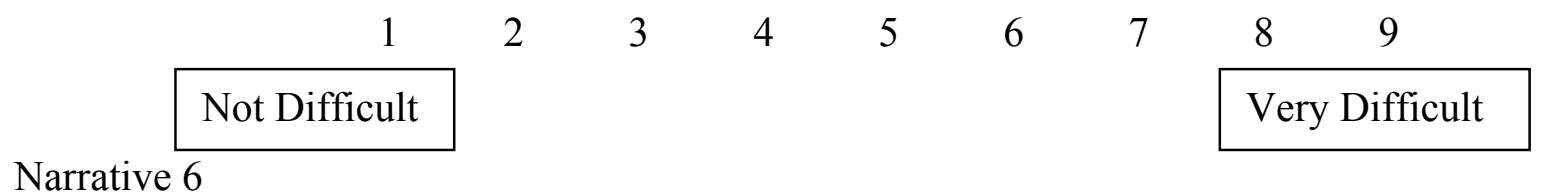

How difficult was it to comprehend the story presented?

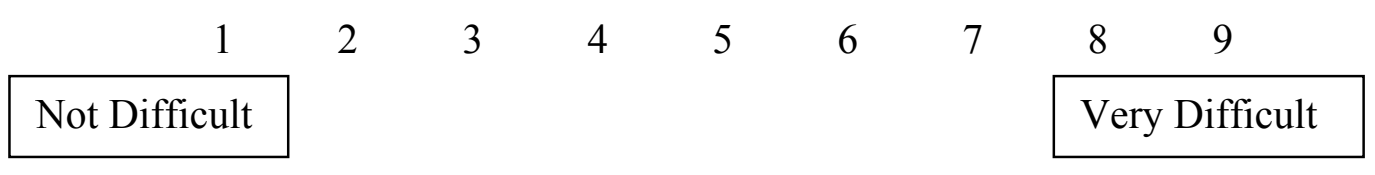




\section{Appendix B}

Episode 1: Exposition

A boy is walking:

Goes down stairs:

Something catches his attention (sees balloon):

Episode 1: Complication

Climbs the pole:

Grabs (retrieves/has/had) balloon:

Episode 1: Resolution

Climbs down (the light pole):

Continues on (down the stairs):

Episode 2: Exposition

Boys (walks/runs) down the (street/home) with balloon:

Lady (mother?) looks out window:

Episode 2: Complication

Lady lets balloon out of the window:

It drifts around:

Episode 2: Resolution

The boy sees the balloon:

Reaches out the window and grabs it (takes it back inside) :

Episode 3: Exposition

Boy walks (away/hides/plays) with no balloon (around corner):

Balloon floats behind (follows/finds) boy:

He grabs it (talks to it/pokes it) :

Episode 3: Complication

Turns around:

Looks for something (lost balloon?):

Episode 3: Resolution

Balloon appears/is found:

Boy (runs/walks) with balloon: 
Episode 4: Exposition

The boy joins a group of (school) children:

Balloon follows him:

Teacher (sees/grabs) at balloon:

Episode 4: Complication

Old man grabs boy:

(Locks/takes) the boy up in another room:

Old man Tries to scare/grab balloon:

Episode 4: Resolution

Old man goes back to the building/door:

Releases the boy:

Balloon is waiting:

Episode 5: Exposition

Woman and boy go up stairs:

Into a building (church?) :

Episode 5: Complication

Balloon follows them into the church:

Episode 5: Resolution

The boy grabs the balloon and runs out of the church:

(chased/followed) by a guard:

woman talks/argues (with guard):

Episode 6: Exposition

The boy's balloon is popped:

(Other/different) people's balloons start leaving:

Episode 6: Complication

(The balloons are) floating down the street (everywhere):

boy is with popped balloon:

Episode 6: Resolution

Balloons (meet/find/discover) boy:

(The balloons) are tied(grabbed) :

The boy rides the balloon into the sky: 
Figure 1
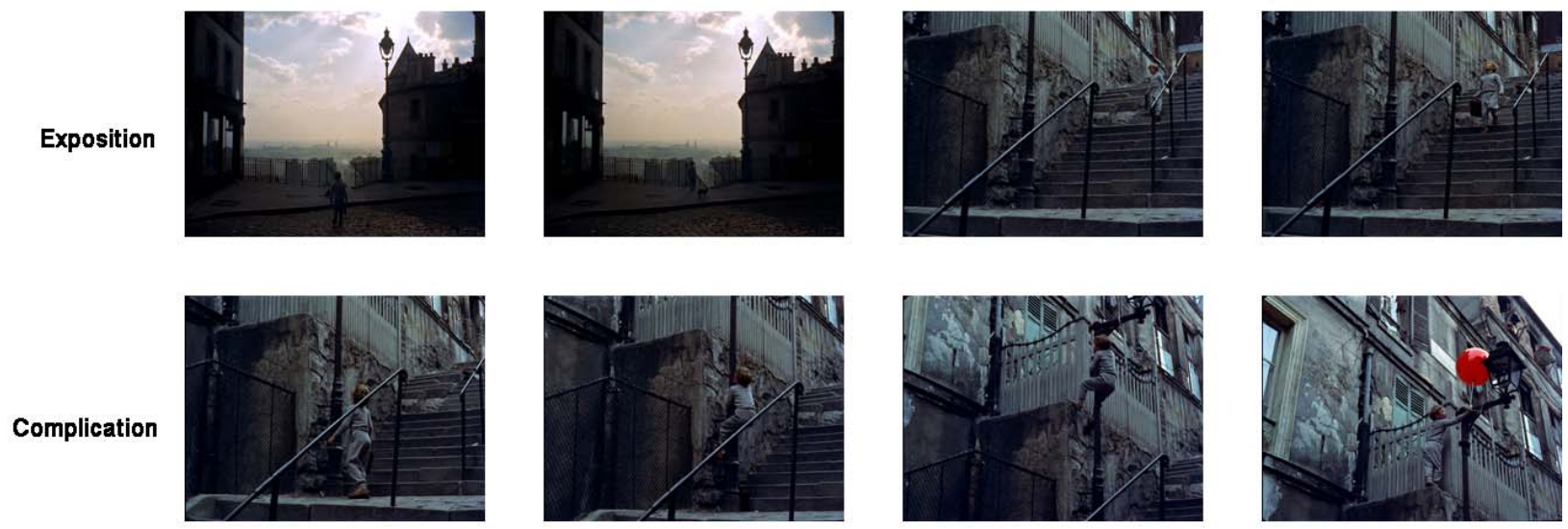

Resolution
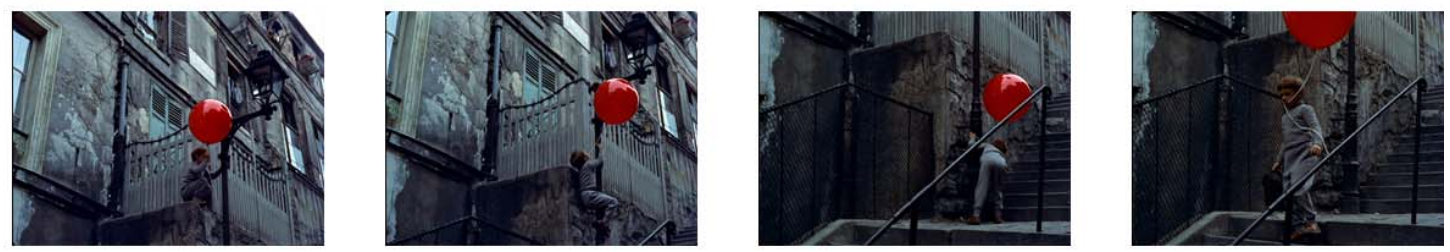
Figure 2

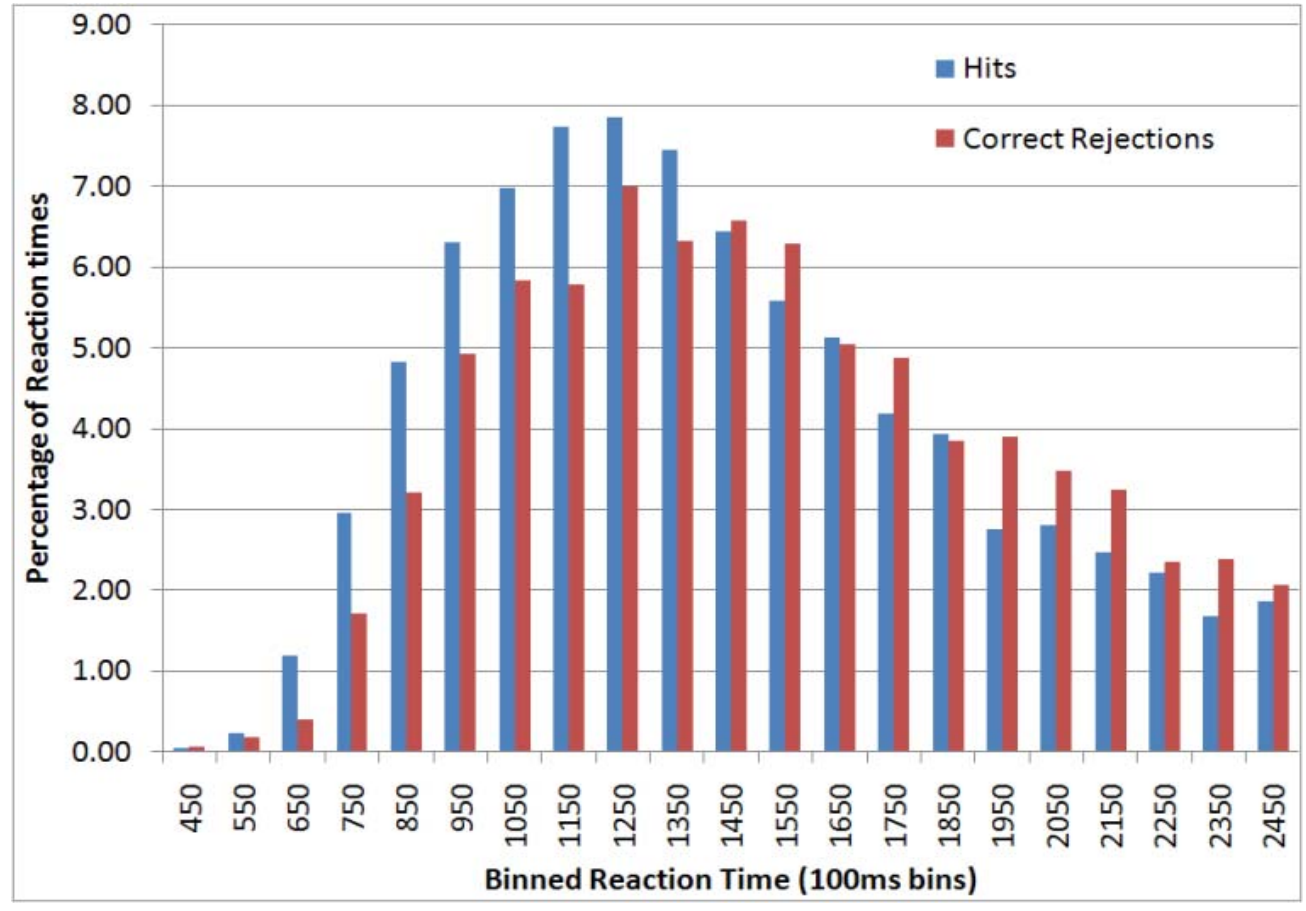


Figure 3

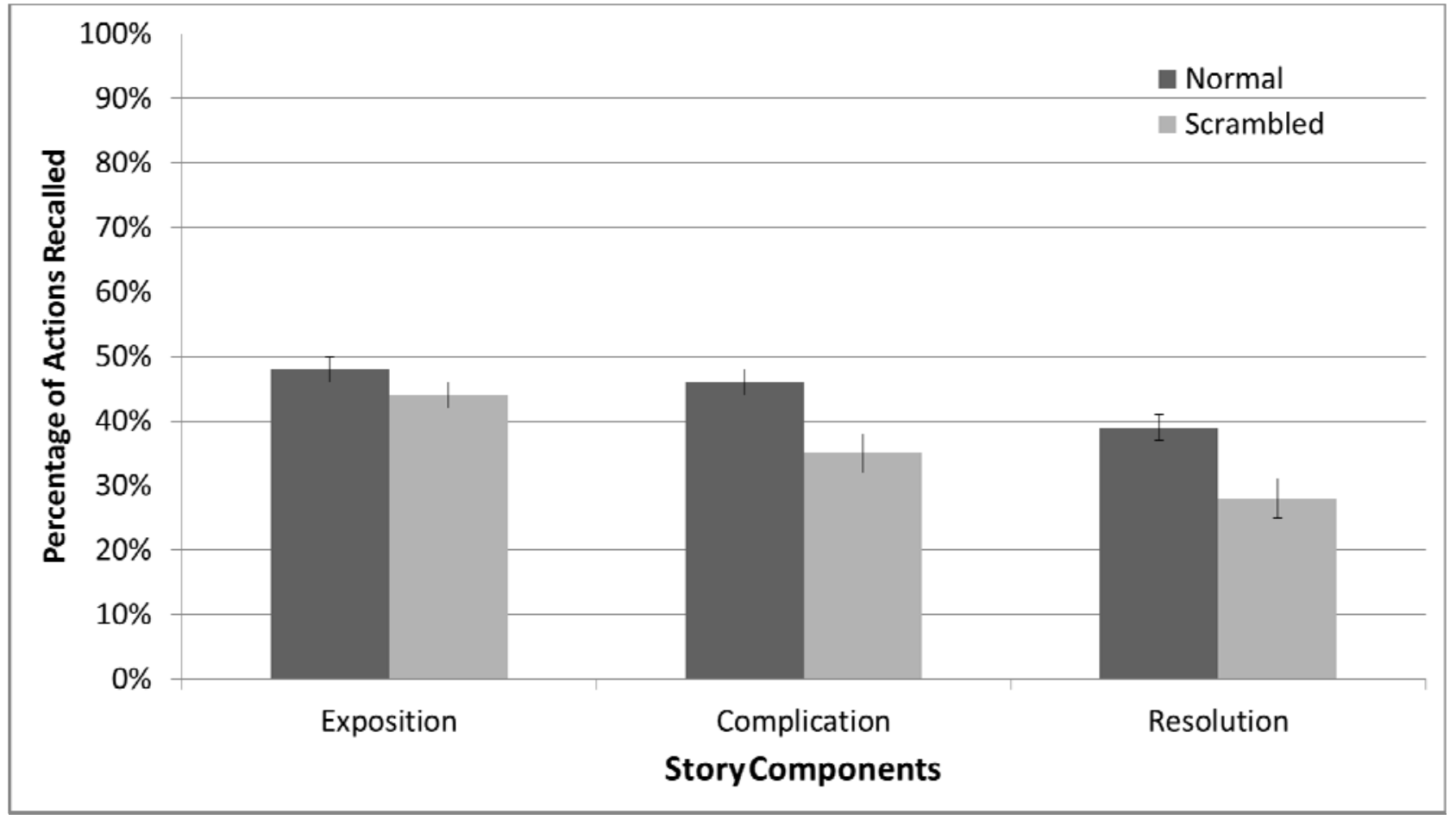


Figure 4
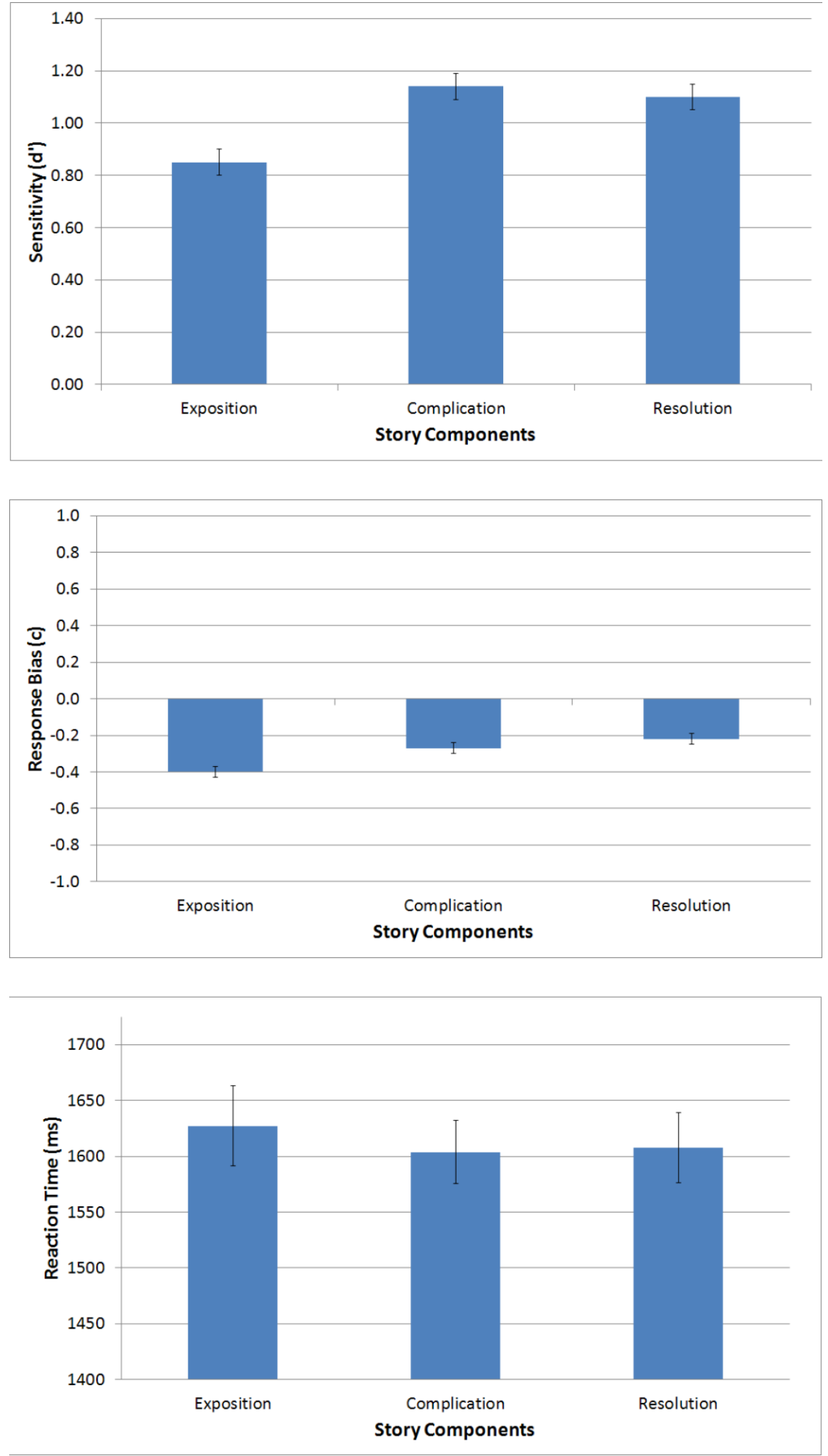
Figure 5

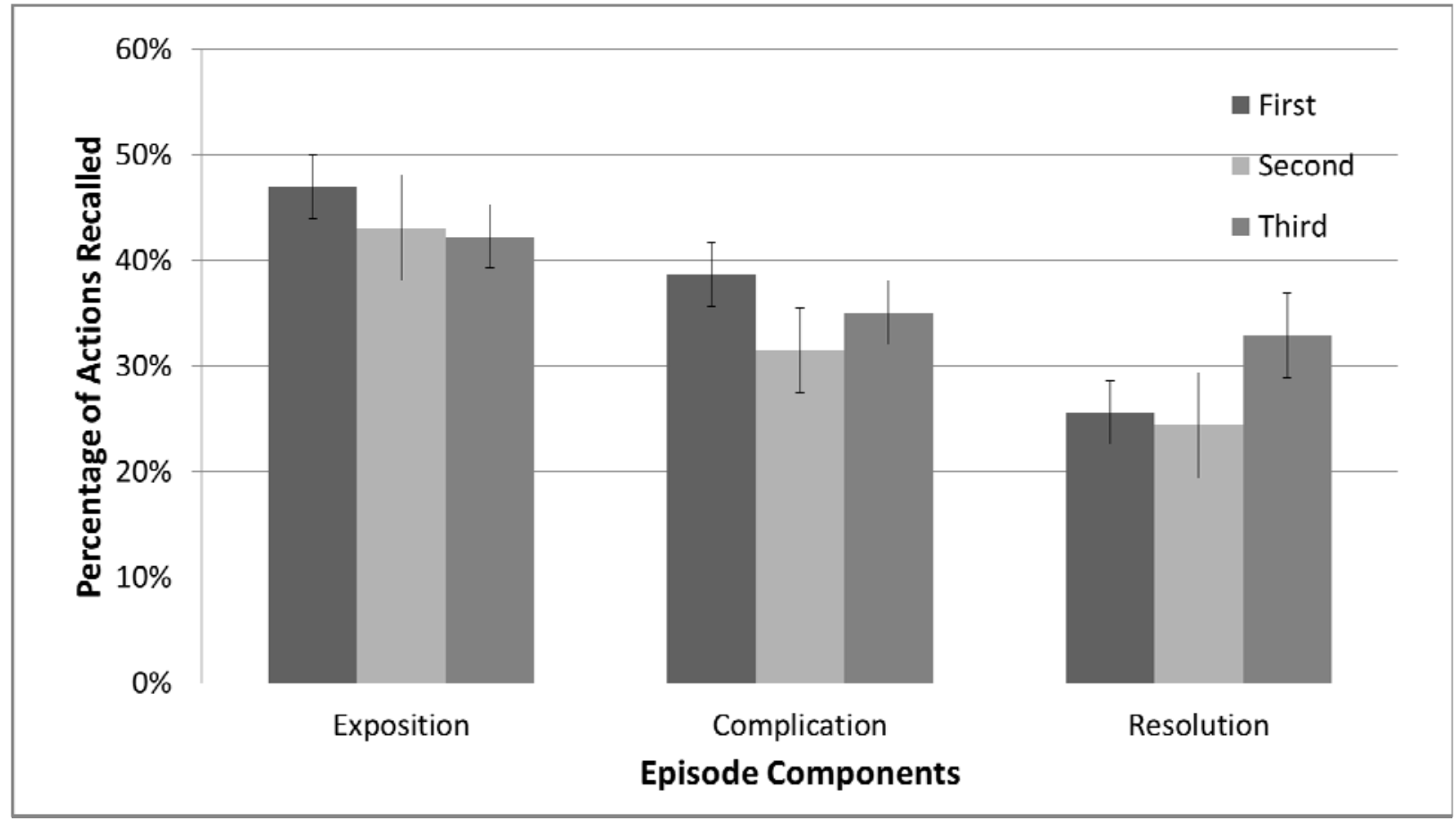


Figure 6
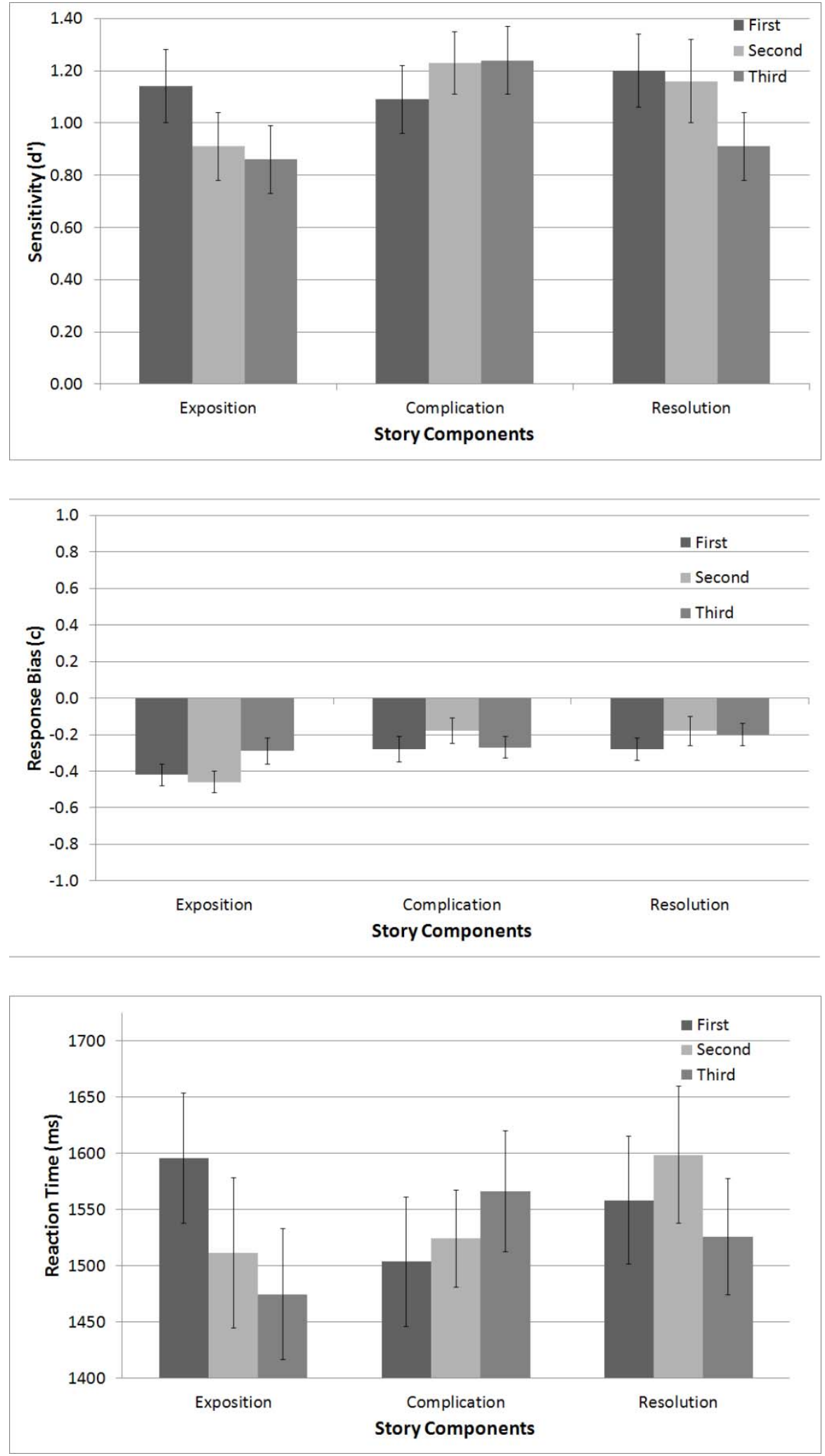
Figure 7
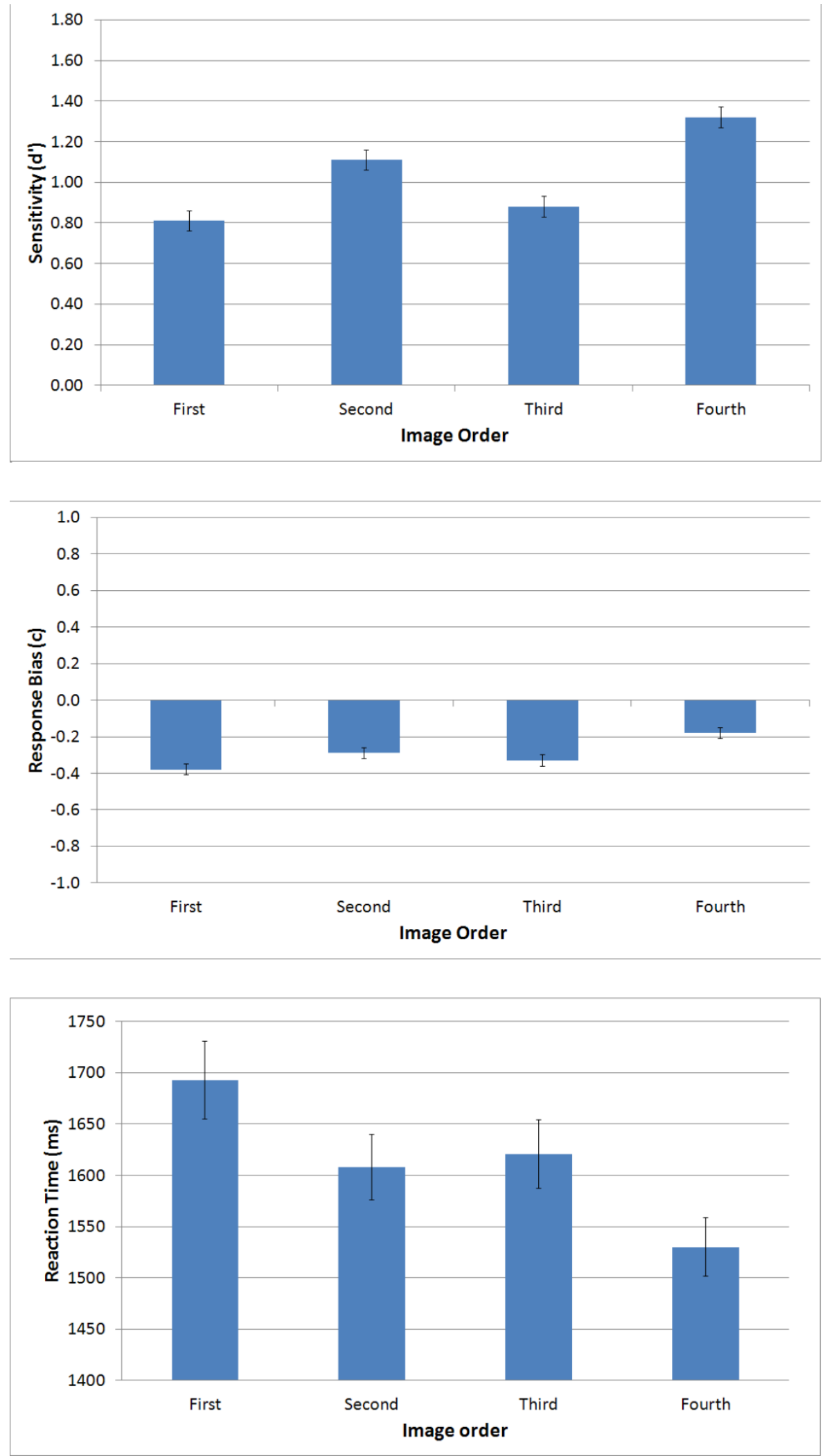
Figure 8
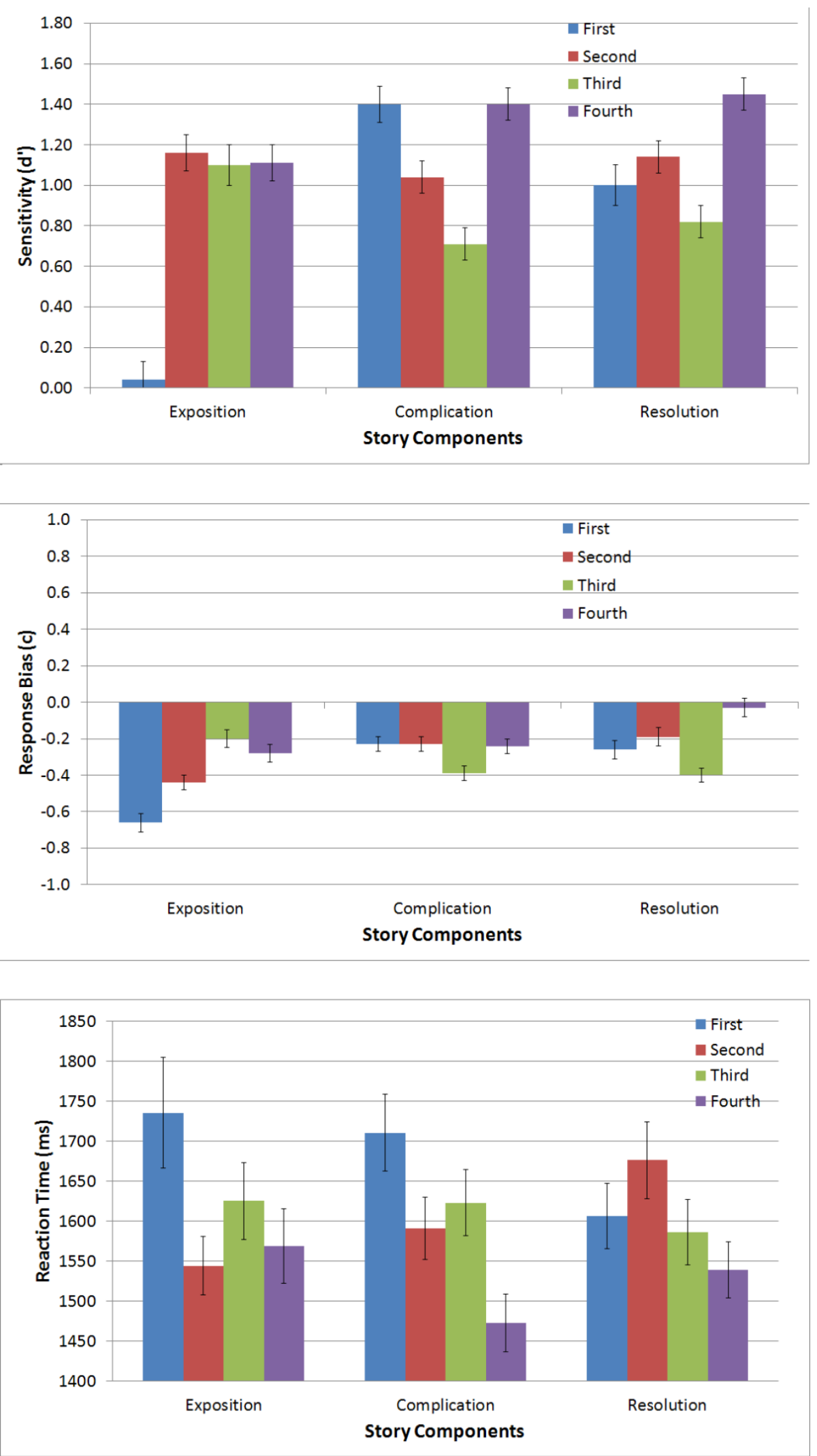
Figure 9

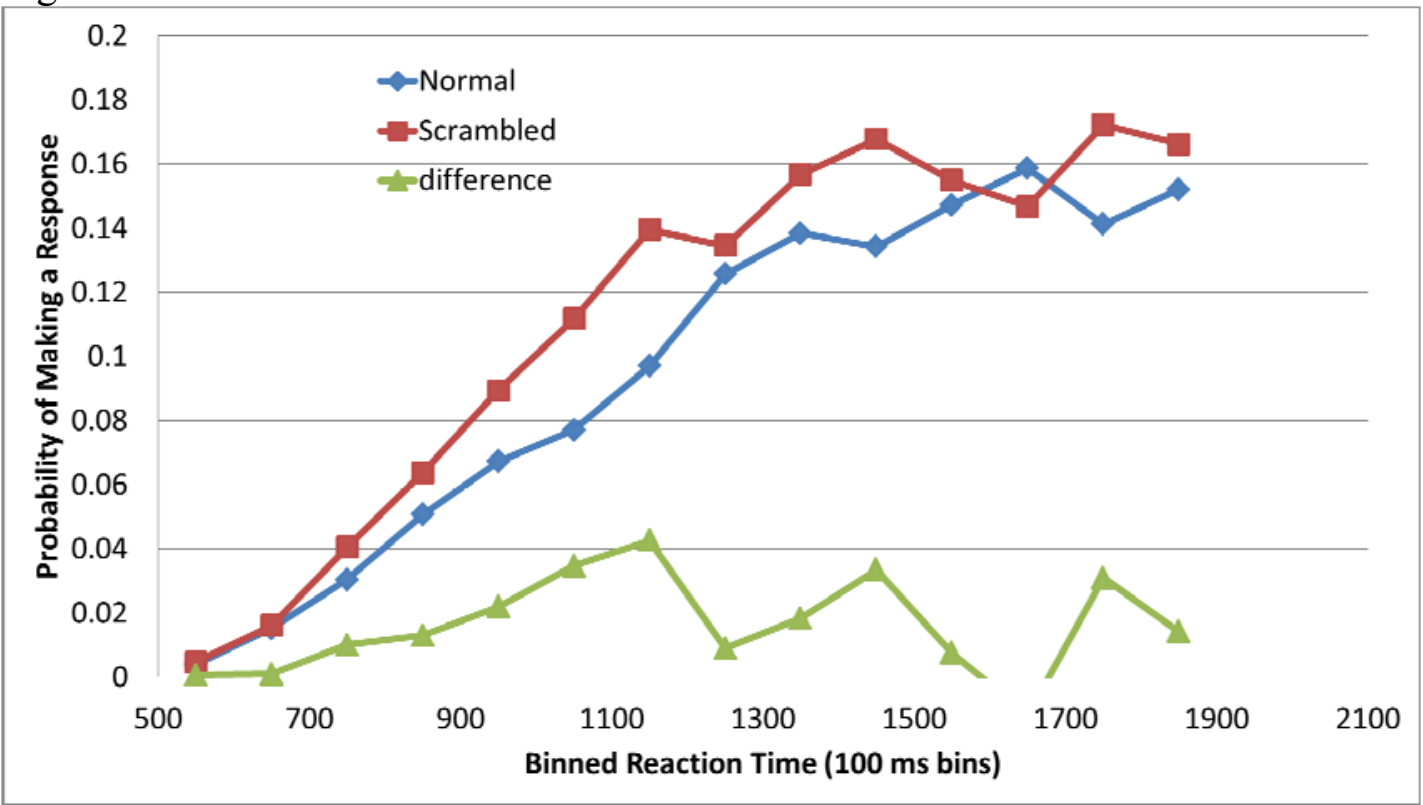




\section{Table 1.}

Descriptive Statistics for the Percentage of Actions Recalled from Each Episode Component by Episode Component Order.

\begin{tabular}{|c|c|c|c|c|c|c|}
\hline \multirow[b]{3}{*}{ Story Order } & \multicolumn{6}{|c|}{ Episode Component } \\
\hline & \multicolumn{2}{|c|}{ Exposition } & \multicolumn{2}{|c|}{ Complication } & \multicolumn{2}{|c|}{ Resolution } \\
\hline & $\underline{\mathrm{M}}$ & $\underline{\mathrm{SD}}$ & $\underline{\mathrm{M}}$ & $\underline{\mathrm{SD}}$ & $\underline{\mathrm{M}}$ & $\underline{\mathrm{SD}}$ \\
\hline Normal & 0.48 & 0.12 & $0.46_{\mathrm{a}}$ & 0.14 & $0.39_{b}$ & 0.18 \\
\hline Scrambled & 0.44 & 0.15 & $0.35_{\mathrm{a}}$ & 0.20 & $0.28_{b}$ & 0.14 \\
\hline Main Effect & $0.46_{\mathrm{c}, \mathrm{d}}$ & 0.13 & $0.41_{\mathrm{c}}$ & 0.17 & $0.34_{d}$ & 0.17 \\
\hline
\end{tabular}

Note. Means sharing subscripts are significantly different. 
Table 2.

Comparisons of Episode Component Recall

\begin{tabular}{lccc}
\hline Effect & df & $F$ & $p$ \\
\hline $\begin{array}{l}\text { Exposition vs. } \\
\text { Complication }\end{array}$ & 1,184 & 12.81 & $<.001$ \\
$\begin{array}{l}\text { Exposition vs. } \\
\text { Resolution }\end{array}$ & 1,184 & 71.18 & $<.001$ \\
$\begin{array}{l}\text { Complication vs. } \\
\text { Resolution }\end{array}$ & 1,184 & 23.64 & $<.001$ \\
\hline
\end{tabular}


Table 3.

Comparisons of Episode Component Recall for the Normal and Scrambled Conditions

\begin{tabular}{|c|c|c|c|}
\hline Effect & $\mathrm{df}$ & $F$ & $p$ \\
\hline \multicolumn{4}{|c|}{ Normal Condition } \\
\hline $\begin{array}{l}\text { Exposition vs. } \\
\text { Complication }\end{array}$ & 1,184 & 1.00 & .32 \\
\hline $\begin{array}{l}\text { Exposition vs. } \\
\text { Resolution }\end{array}$ & 1,184 & 20.45 & $<.001$ \\
\hline $\begin{array}{l}\text { Complication vs. } \\
\text { Resolution }\end{array}$ & 1,184 & 12.27 & $<.001$ \\
\hline \multicolumn{4}{|c|}{ Scrambled Condition } \\
\hline $\begin{array}{l}\text { Exposition vs. } \\
\text { Complication }\end{array}$ & 1,184 & 15.18 & $<.001$ \\
\hline $\begin{array}{l}\text { Exposition vs. } \\
\text { Resolution }\end{array}$ & 1,184 & 53.00 & $<.001$ \\
\hline $\begin{array}{l}\text { Complication vs. } \\
\text { Resolution }\end{array}$ & 1,184 & 11.45 & $<.001$ \\
\hline
\end{tabular}


Table 4.

Episode Component Descriptive Statistics for Sensitivity, Bias, and Reaction Time

\begin{tabular}{|c|c|c|c|c|c|c|}
\hline \multirow[b]{2}{*}{$\begin{array}{l}\text { Episode } \\
\text { Component }\end{array}$} & \multicolumn{2}{|c|}{ Sensitivity (d') } & \multicolumn{2}{|c|}{ Bias (c) } & \multicolumn{2}{|c|}{ Reaction Time (ms) } \\
\hline & $\underline{\mathrm{M}}$ & $\underline{\mathrm{SD}}$ & $\underline{\mathrm{M}}$ & $\underline{\mathrm{SD}}$ & $\underline{\mathrm{M}}$ & $\underline{\mathrm{SD}}$ \\
\hline Exposition & $0.85_{\mathrm{a}, \mathrm{b}}$ & 0.52 & $-0.40_{a, b}$ & 0.26 & 1627.31 & 350.10 \\
\hline Complication & $1.14_{\mathrm{a}}$ & 0.44 & $-0.27 \mathrm{a}$ & 0.26 & 1603.68 & 273.63 \\
\hline Resolution & $1.10_{\mathrm{b}}$ & 0.50 & $-0.22_{b}$ & 0.29 & 1607.55 & 303.51 \\
\hline
\end{tabular}

Note. Means in a column sharing subscripts are significantly different. For the bias measure, positive means indicate a "New scene" response bias, while negative means indicate an "old scene" response bias in the recognition test. 
Table 5.

Comparisons of Episode Component Recognition Memory (d')

\begin{tabular}{lccc}
\hline Effect & df & $F$ & $p$ \\
\hline $\begin{array}{l}\text { Exposition vs. } \\
\text { Complication }\end{array}$ & 1,184 & 4.76 & .03 \\
$\begin{array}{l}\text { Exposition vs. } \\
\text { Resolution }\end{array}$ & 1,184 & 3.63 & .06 \\
$\begin{array}{l}\text { Complication vs. } \\
\text { Resolution }\end{array}$ & 1,184 & 0.08 & .78 \\
\hline
\end{tabular}


Table 6.

Comparisons of Episode Component Bias (c)

\begin{tabular}{lccc}
\hline Effect & df & $F$ & $p$ \\
\hline $\begin{array}{l}\text { Exposition vs. } \\
\text { Complication }\end{array}$ & 1,184 & 4.29 & .04 \\
$\begin{array}{l}\text { Exposition vs. } \\
\text { Resolution }\end{array}$ & 1,184 & 7.97 & .006 \\
$\begin{array}{l}\text { Complication vs. } \\
\text { Resolution }\end{array}$ & 1,184 & 0.57 & .45 \\
\hline
\end{tabular}


Table 7.

Comparing Recall Memory in the Scrambled Condition

\begin{tabular}{lccc}
\hline Effect & df & $F$ & $p$ \\
\hline $\begin{array}{l}\text { Exposition vs. } \\
\text { Complication }\end{array}$ & 1,82 & 4.72 & .03 \\
$\begin{array}{l}\text { Exposition vs. } \\
\text { Resolution }\end{array}$ & 1,82 & 15.83 & .001 \\
$\begin{array}{l}\text { Complication vs. } \\
\text { Resolution }\end{array}$ & 1,82 & 3.25 & .08 \\
\hline
\end{tabular}


Table 8.

Comparing Episode Component Recognition Memory (d') for the Scrambled Condition

\begin{tabular}{lccc}
\hline Effect & df & $F$ & $p$ \\
\hline $\begin{array}{l}\text { Exposition vs. } \\
\text { Complication }\end{array}$ & 1,82 & 1.96 & .17 \\
$\begin{array}{l}\text { Exposition vs. } \\
\text { Resolution }\end{array}$ & 1,82 & 0.61 & .44 \\
$\begin{array}{l}\text { Complication vs. } \\
\text { Resolution }\end{array}$ & 1,82 & 0.38 & .54 \\
\hline
\end{tabular}




\section{Table 9.}

Sensitivity (d') Descriptive Statistics for the Component Order for each Episode Component

\begin{tabular}{|c|c|c|c|c|c|c|}
\hline \multirow[b]{3}{*}{$\begin{array}{l}\text { Component } \\
\text { Order }\end{array}$} & \multicolumn{6}{|c|}{ Episode Component } \\
\hline & \multicolumn{2}{|c|}{ Exposition } & \multicolumn{2}{|c|}{ Complication } & \multicolumn{2}{|c|}{ Resolution } \\
\hline & $\underline{\mathrm{M}}$ & $\underline{\mathrm{SD}}$ & $\underline{\mathrm{M}}$ & $\underline{\mathrm{SD}}$ & $\underline{\mathrm{M}}$ & $\underline{\mathrm{SD}}$ \\
\hline First & 1.14 & 0.91 & 1.09 & 0.86 & 1.20 & 0.86 \\
\hline Second & 0.91 & 0.85 & 1.23 & 0.75 & 1.16 & 0.85 \\
\hline Third & 0.86 & 0.90 & 1.24 & 1.03 & 0.91 & 0.87 \\
\hline
\end{tabular}


Table 10.

Reaction Time (ms) Descriptive Statistics for the Component Order for each Episode Component

\begin{tabular}{|c|c|c|c|c|c|c|}
\hline \multirow[b]{3}{*}{$\begin{array}{l}\text { Component } \\
\text { Order }\end{array}$} & \multicolumn{6}{|c|}{ Episode Component } \\
\hline & \multicolumn{2}{|c|}{ Exposition } & \multicolumn{2}{|c|}{ Complication } & \multicolumn{2}{|c|}{ Resolution } \\
\hline & $\underline{\mathrm{M}}$ & $\underline{\mathrm{SD}}$ & $\underline{\mathrm{M}}$ & $\underline{\mathrm{SD}}$ & $\underline{\mathrm{M}}$ & $\underline{\mathrm{SD}}$ \\
\hline First & 1595.75 & 374.12 & 1503.80 & 371.67 & 1558.33 & 367.46 \\
\hline Second & 1511.38 & 432.42 & 1524.42 & 280.24 & 1598.68 & 394.62 \\
\hline Third & 1474.83 & 377.94 & 1566.28 & 347.83 & 1525.96 & 334.33 \\
\hline Main Effect & 1527.32 & 305.73 & 1531.50 & 252.90 & 1560.99 & 295.59 \\
\hline
\end{tabular}


Table 11.

Sensitivity (d') Descriptive Statistics for the Serial Image Order of each Episode Component

\begin{tabular}{|c|c|c|c|c|c|c|}
\hline \multirow[b]{3}{*}{ Image Position } & \multicolumn{6}{|c|}{ Episode Component } \\
\hline & \multicolumn{2}{|c|}{ Exposition } & \multicolumn{2}{|c|}{ Complication } & \multicolumn{2}{|c|}{ Resolution } \\
\hline & $\underline{\mathrm{M}}$ & $\underline{\mathrm{SD}}$ & $\underline{\mathrm{M}}$ & $\underline{\mathrm{SD}}$ & $\underline{\mathrm{M}}$ & $\underline{\mathrm{SD}}$ \\
\hline First & $0.04_{a}$ & 0.88 & $1.40_{\mathrm{a}}$ & 0.87 & 1.00 & 0.92 \\
\hline Second & $1.16_{\mathrm{a}}$ & 0.88 & $1.04_{\mathrm{a}, \mathrm{b}}$ & 0.80 & 1.14 & 0.77 \\
\hline Third & 1.10 & 0.96 & $0.71_{b, c}$ & 0.76 & $0.82_{\mathrm{a}}$ & 0.82 \\
\hline Fourth & 1.11 & 0.89 & $1.40_{\mathrm{c}}$ & 0.73 & $1.45_{\mathrm{a}}$ & 0.80 \\
\hline
\end{tabular}

Note. Means in a column sharing subscripts are significantly different. 
Table 12.

Comparing Recognition Memory (d") of the Serial Image Order for each Episode Component

\begin{tabular}{|c|c|c|c|}
\hline Effect & $\mathrm{df}$ & $F$ & $p$ \\
\hline \multicolumn{4}{|c|}{ Exposition } \\
\hline First vs. Second & 1,552 & 90.38 & $<.001$ \\
\hline Second vs. Third & 1,552 & 0.36 & .13 \\
\hline Third vs. Fourth & 1,552 & 0.08 & .28 \\
\hline \multicolumn{4}{|c|}{ Complication } \\
\hline First vs. Second & 1,552 & 7.86 & .005 \\
\hline Second vs. Third & 1,552 & 7.51 & .006 \\
\hline Third vs. Fourth & 1,552 & 32.48 & $<.001$ \\
\hline \multicolumn{4}{|c|}{ Resolution } \\
\hline First vs. Second & 1,552 & 1.43 & .23 \\
\hline Second vs. Third & 1,552 & 7.42 & .007 \\
\hline Third vs. Fourth & 1,552 & 28.38 & $<.001$ \\
\hline
\end{tabular}




\section{Table 13.}

Reaction Time (ms) Descriptive Statistics for the Serial Image Order of each Episode Component

\begin{tabular}{|c|c|c|c|c|c|c|}
\hline \multirow[b]{3}{*}{ Image Position } & \multicolumn{6}{|c|}{ Episode Component } \\
\hline & \multicolumn{2}{|c|}{ Exposition } & \multicolumn{2}{|c|}{ Complication } & \multicolumn{2}{|c|}{ Resolution } \\
\hline & $\underline{\mathrm{M}}$ & $\underline{\mathrm{SD}}$ & $\underline{\mathrm{M}}$ & $\underline{\mathrm{SD}}$ & $\underline{\mathrm{M}}$ & $\underline{\mathrm{SD}}$ \\
\hline First & $1746.47_{\mathrm{a}}$ & 670.49 & $1715.58_{\mathrm{a}}$ & 463.75 & 1616.17 & 401.68 \\
\hline Second & 1549.28 & 355.73 & 1596.33 & 377.05 & 1677.70 & 461.02 \\
\hline Third & 1637.86 & 477.86 & $1630.91_{b}$ & 399.75 & 1593.80 & 395.87 \\
\hline Fourth & $1575.61_{\mathrm{a}}$ & 451.13 & $1471.69_{\mathrm{a}, \mathrm{b}}$ & 345.67 & 1542.52 & 338.34 \\
\hline
\end{tabular}

Note. Means in a column sharing subscripts are significantly different. 
Table 14

Comparisons of Recognition Memory Reaction Time for the Serial Image order in each Episode Component

\begin{tabular}{|c|c|c|c|}
\hline Effect & df & $F$ & $p$ \\
\hline \multicolumn{4}{|c|}{ Exposition } \\
\hline First vs. Second & 1,552 & 13.11 & $<.001$ \\
\hline Second vs. Third & 1,552 & 2.34 & .13 \\
\hline Third vs. Fourth & 1,552 & 1.15 & .28 \\
\hline First vs. Fourth & 1,552 & 10.00 & .002 \\
\hline \multicolumn{4}{|c|}{ Complication } \\
\hline First vs. Second & 1,552 & 5.13 & .02 \\
\hline Second vs. Third & 1,552 & 0.37 & .54 \\
\hline Third vs. Fourth & 1,552 & 8.12 & .0045 \\
\hline First vs. Fourth & 1,552 & 20.29 & $<.001$ \\
\hline \multicolumn{4}{|c|}{ Resolution } \\
\hline First vs. Second & 1,552 & 1.74 & .19 \\
\hline Second vs. Third & 1,552 & 2.91 & .09 \\
\hline Third vs. Fourth & 1,552 & 0.80 & .37 \\
\hline First vs. Fourth & 1,552 & 1.65 & .20 \\
\hline
\end{tabular}

